\title{
Vibration and Buckling of Skew Plates Under Linearly Varying Edge Compression
}

\author{
Abhinav Kumar, Sarat Kumar Panda and Sekhar Chandra Dutta \\ Department of Civil Engineering, Indian School of Mines, Dhanbad-826004, India. \\ Rajesh Kumar \\ Department of Civil Engineering, Indian Institute of Technology Kharagpur, Kharagpur-721302, India.
}

\begin{abstract}
(Received 21 February 2016; accepted 30 June 2016)
Pre-buckling vibration and buckling behaviour of composite skew plates subjected to linearly varying in-plane edge loading with different boundary conditions are studied. The total energy functional of the skew plate mapped from physical domain to computational domain over which a set of orthonormal polynomials satisfying the essential boundary conditions is generated by Gram-Schmidt orthogonalization process. Using Rayleigh-Ritz method in conjunction with Boundary Characteristics Orthonormal Polynomials, the total energy functional is converted into sets of algebraic equations for static stability problems and ordinary differential equation for free vibration problem. Pre-buckling vibration frequencies of the stressed skew plate are obtained by solving associated linear eigen value problem for free vibration and solution of the eigen value problem for static case results critical buckling load. From different parametric study, it is observed that the pre-buckling vibration frequency and critical buckling load increase with the increase of skew angle and edge restraint.
\end{abstract}

\section{NOMENCLATURE}

$\begin{array}{ll}a, b & \text { Plan-form dimension of plate } \\ a / b & \text { Aspect ratio of plate } \\ \bar{N}_{\xi \xi} \text { and } \bar{N}_{\eta \eta} & \text { Applied edge forces along } \xi \text { and } \eta \text { axes } \\ N_{x c r} & \text { Buckling load } \\ u, v & \text { In-plane displacement } \\ w & \text { Out-of-plane displacement } \\ x, y, z & \text { Cartesian coordinates } \\ \varphi_{1}, \varphi_{2} & \text { Rotations of the reference surface } \\ M_{x x}, M_{y y}, M_{x y} & \text { about the } \xi_{2} \text { and } \xi_{1} \text { axes } \\ N_{x x}, N_{y y}, N_{x y} & \text { Foment resultant } \\ P_{x x}, P_{y y}, P_{x y} & \text { Additional moment resultant } \\ S & \text { Strain energy } \\ \Phi & \text { Stress function } \\ N_{s} & \text { Static load factor } \\ N_{t} & \text { Dynamic load factor } \\ S^{m} & \text { Membrane energy } \\ S^{b} & \text { Bending Energy } \\ S^{a b} & \text { Additional bending energy } \\ S^{s} & \text { Shear energy } \\ \psi & \text { Skew angle } \\ \omega^{*} & \text { Non-dimensional fundamental frequency } \\ \omega & \text { Fundamental frequency } \\ \Pi & \text { Total potential energy } \\ & \end{array}$

\section{INTRODUCTION}

Composite skew plates are widely used as structural elements in many engineering applications such as aircraft wings, marine structures, ship structures, and bridges. These composite plates are generally preferred because of their high strengthto-weight ratio and ability to be moulded according to any desired orientation. In a complex structural system, the loading coming on it may not be uniform. For a few simple examples, the web of an I- beam, aircraft wings, and the stiffened plates in ship structures are generally subjected to other than uniform in-plane loading. There is literature available on the stability of rectangular plates subjected to non-uniform in-plane loading. But so far as authors' knowledge, studies on the pre-buckling vibration and static stability of a skew plate under non-uniform or linearly varying in-plane loading is not available in the open literature.

Literatures are available on stability and vibration studies of skew plates under uniform in-plane loading. Durvasula worked on buckling problems of simply supported skew plates with in-plane stresses represented in terms of orthogonal components using the Rayleigh-Ritz method (RRM), employing a double Fourier sine series in oblique coordinates. ${ }^{1}$ Liew and Lam used two-dimensional orthogonal plate functions as an admissible deflection function for the study of the flexural vibration of skew plates with the RRM. ${ }^{2}$ Authors followed the Gram-Schmidt orthogonalization process to generate twodimensional orthogonal plate functions. Wang et al. performed the buckling analysis using the RRM with pb-2 Ritz functions, which consist of the product of a two-dimensional polynomial function and a basic function. ${ }^{3}$ The first five frequencies have been determined for the transverse vibration of a rectangular or a skew plate under different boundary conditions by using boundary characteristic orthogonal polynomials (BCOP) in two variables by Singh and Chakraverty. ${ }^{4}$ Wang presented a B-spline RRM based on first-order shear deformation (FOSD) plate theory for a buckling study of composite skew plates. ${ }^{5}$ Mizusawa et al. presented a method for evaluating the buckling of stiffened skew plates by using theRRM with B-spline functions as co-ordinate functions. ${ }^{6}$ Taj and Chakrabarti studied the static and dynamic analysis of functionally-graded skew plates by using the finite element method (FEM) based on the third-order shear deformation (TOSD) theory. ${ }^{7}$ Kitipornchai et al. followed the principle of stationary total potential en- 
ergy in conjunction with the pb-2 RRM for a buckling analysis of skew plates of various aspect ratios, skew angles, and boundary conditions. ${ }^{8}$ Farag and Ashour used the Kantorovich method, a fast-converging, semi-analytical method for a vibration analysis of thin orthotropic skew plates. ${ }^{9}$ In this method, the finite strip method is modified to reduce the complexity of the problem. Ganapathi and Prakash studied the thermal buckling of functionally-graded simply supported skew plates using FOSD theory with the FEM. ${ }^{10}$ The authors considered linear and nonlinear temperature variation across the thickness. Wang et al. proposed a modified version of the differential quadrature method (DQM) for analysing the static stability of different skew plates. ${ }^{11}$ Numerical results indicate that the modified DQM is faster in convergence study and gives better results than the old DQM version. Daripa and Singha worked on the stability characteristics of composite skew plates subjected to in-plane compressive load using the shear deformable finite element approach. ${ }^{12}$ The effect of pre-buckling stresses at the corner regions of isotropic and composite skew plates has been studied. Karami et al. followed the DQM for static, free vibration, and stability analysis of thin trapezoidal composite skew plates. ${ }^{13}$ The authors used the general transformation scheme for transferring the variation of the variables in the computational domain to the physical domain and vice versa. Zhou and Zheng employed the moving least square (MLS) method for analysing the vibration characteristic of skew plates with large skew angles. ${ }^{14}$ The MLS technique in conjunction with the Ritz method is applied to derive the governing eigenvalue equation for the skew plate. The boundary conditions of the plate are satisfied through a point substitution technique that makes the MLS-Ritz trial function satisfy the essential boundary conditions along the plate edges. Singha and Ganapathi investigated the large amplitude free flexural vibration of thin laminated composite skew plates within the frame work of the FEM. ${ }^{15}$ Lagrange's equations of motion and von Karman's geometric nonlinearity is used to derive the governing differential equation. Taj and Chakrabarti worked on the static and dynamic analysis of functionally graded material (FGM) skew plates under mechanical loading by using the FEM. The authors employed the FEM formulation based on the TOSD theory. ${ }^{7}$ Eftekhari and Jafari proposed modified mixed Ritzdifferential quadrature (Ritz-DQ) methodology for a vibration and buckling study of rectangular skew plates. ${ }^{16}$ All the natural boundary conditions are exactly implemented by modified mixed Ritz-DQ formulation. The method resulted in accurate solutions for the natural frequencies of thick rectangular plates involving adjacent free edges and skew plates using a small number Ritz terms and DQ sampling points. Vibration analysis of skew plates with different boundary conditions by using the new version of the DQM is investigated by Wang et al. (2014). ${ }^{17}$ The authors observed good convergence of frequency for the simply supported skew plate with a large skew angle.

There are many research articles available in open literature on the static stability analysis of rectangular plates subjected to non-uniform in-plane loading or linearly varying in-plane loading. Leissa and Kang (2002) and Kang and Leissa (2001, 2005) presented exact solutions for a plate subjected to different types of linearly varying in-plane loading with different boundary conditions. ${ }^{18-20}$ Zhong and Gu presented the exact solution of the buckling of moderately thick simply sup- ported plates with symmetric cross-ply laminates under different linearly varying in-plane edge loads. ${ }^{21}$ The authors used the FOSD theory for obtaining the buckling load for various aspect ratios of the composite plates. Lai and Xiang presented the discrete singular convolution method for solving buckling and vibration problems of rectangular plates with elastically restrained edges and linearly varying uni-axial in-plane loading. ${ }^{22}$ Authors presented accurate buckling and vibration solutions of plates having two opposite edges elastically restrained and the other two sides clamped. Lopatin and Morozov presented the analytical solution of the buckling problem for an orthotropic rectangular plate having two parallel edges simply supported, one edge clamped, and the remaining edge free (the SSCF plate). ${ }^{23}$ The plate considered is subjected to a linearly varying in-plane load, and the solution technique involves the Kantorovich procedure in conjunction with the generalised Galerkin method. The buckling problems are solved for isotropic and orthotropic plates with various aspect ratios. The buckling of thin rectangular plates with nonlinearly distributed loadings along two opposite plate edges is analysed using the DQM by Wang et al. $(2006,2007){ }^{24,25}$ The authors first solved the plane elasticity problem to obtain the distribution of in-plane stresses, and then the buckling problem was solved. The DQM was proved an accurate and computationally efficient numerical method by Wang et al. (2006). ${ }^{26}$ Authors used this method to study the vibration and buckling of an SS-C-SS-C rectangular plate loaded by linearly varying in-plane stresses. ${ }^{26}$ Tang and Wang analysed symmetrically laminated rectangular plates with parabolic distributed in-plane compressive loadings along two opposite edges using the RRM in conjunction with the classical laminated plate theory. ${ }^{27}$ Stress functions satisfying all stress boundary conditions are constructed based on the Chebyshev polynomials. It was observed that double sine series displacement for simply supported symmetrically laminated plates overestimate buckling loads. Recently, Kumar et al. studied the parametric resonance of a composite skew plate under non-uniform in-plane loading using the RRM in conjunction with BCOP functions. ${ }^{28}$

So far, to the best of the authors' knowledge, there is no work available in open literature on the pre-buckling vibration and buckling of composite skew plates based on higher-order shear deformation theory (HSDT) under non-uniform or linearly varying in-plane loading. In the present investigation, the pre-buckling vibration and buckling of composite skew plates subjected to linearly varying in-plane loading is considered for different boundary conditions. The linearly varying in-plane edge compression causes same linearly varying stress in the skew plate stress. The total energy functional is formulated by using the in-plane stress distribution. The total energy functional is transformed from the physical domain to the computational domain using transformation relations. The orthonormal polynomials are generated by using the Gram-Schmidt orthogonalization process, which satisfies the essential boundary conditions of skew plates in computational domain. Using the RRM in conjunction with BCOPs, the total energy functional is converted into sets of algebraic equations for the static stability problem and into ordinary differential equations for the free vibration problem. Pre-buckling vibration frequencies of the stressed skew plate are obtained by solving the associated linear eigenvalue problem for free vibration. The solution of theeigenvalue problem for the static case results in the critical 


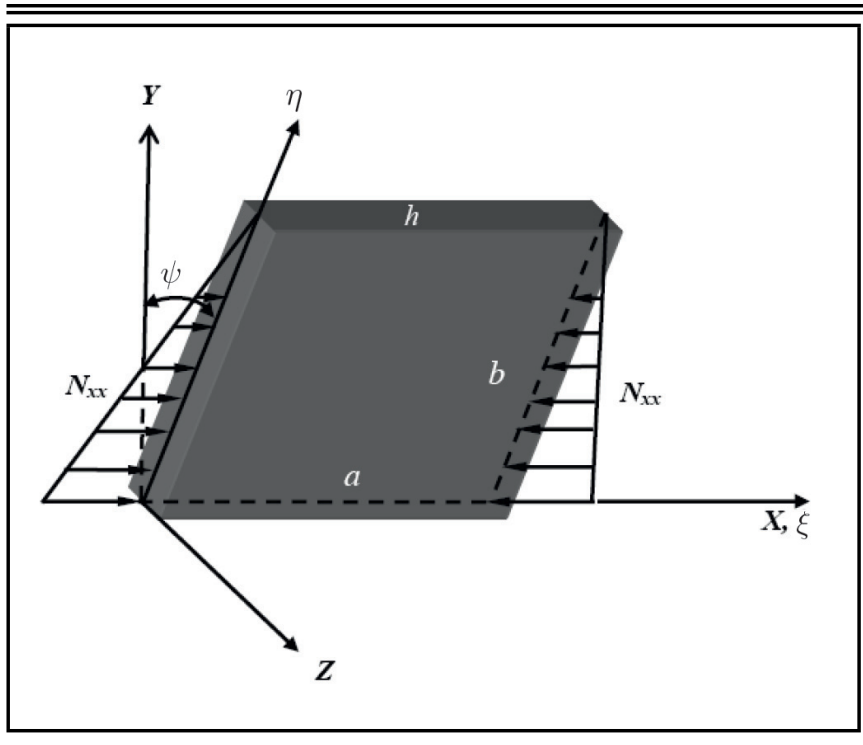

Figure 1a. Geometry and linearly varying in-plane loading of the skew plate.

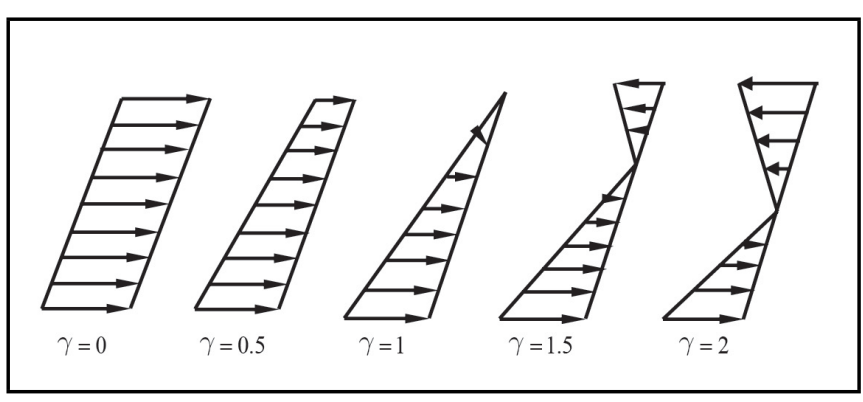

Figure 1b. Various types of linearly varying loadings.

buckling load. The effects of skew angles, boundary conditions, shear deformations, aspect ratios, and loadings on the pre-buckling vibration and buckling behaviour of composite skew plates are presented.

\section{FORMULATION}

In this study, composite skew plates are analysed having length " $a$," width " $b$," and " $l_{n}$ " layers of equal thickness laminates as shown in Fig. 1a. The $z$-axis is in the direction perpendicular to the $\xi-\eta$ plane. Different types of linearly varying inplane edge loadings are considered for finding the pre-buckling frequencies and static stability of the composite skew plate. The linearly varying in-plane edge loadings are expressed as: $N_{x x}=N_{0}\left(1-\gamma\left(\frac{\eta}{b}\right)\right)$. For different values of $\gamma$, we obtain different in-plane load distributions: uniform $(\gamma=0)$, trapezoidal $(\gamma=0.5)$, triangular $(\gamma=1)$, partial tension $(\gamma=1.5)$, and in-plane bending $(\gamma=2)$. The linearly varying in-plane loads are shown in Fig. 1b.

Higher-order shear deformation theory (HSDT) is implemented for composite plates as proposed by Reddy and Liu. ${ }^{29}$ In this theory, the displacements of the middle surface are expressed as cubic functions of the thickness coordinate, and the transverse displacement is assumed to be constant through the thickness. For this displacement field, the distribution of transverse shear stress through the thickness is of parabolic variation. The transverse shear stress is zero at both the top and bottom free surface and is maximum at the middle surface. The displacement fields may be written as:

$$
\begin{gathered}
\begin{aligned}
& u(x, y, z, t)=u^{o}(x, y, t)+z \varphi_{1}(x, y, t)+ \\
& z^{3}\left(4 / 3 h^{2}\right)\left[-\varphi_{1}(x, y, t)-w_{, x}^{o}(x, y, t)\right]
\end{aligned} \\
\begin{aligned}
& v(x, y, z, t)=v^{o}(x, y, t)+z \varphi_{2}(x, y, t)+ \\
& z^{3}\left(4 / 3 h^{2}\right)\left[-\varphi_{2}(x, y, t)-w_{, y}^{o}(x, y, t)\right]
\end{aligned} \\
\begin{aligned}
w(x, y, z, t) & =w^{o}(x, y, t)
\end{aligned}
\end{gathered}
$$

The above displacement fields can be rearranged as Soldatos displayed: ${ }^{30}$

$$
\begin{aligned}
u(x, y, z, t) & =u^{o}(x, y, t)-z w_{, x}^{o}(x, y, t)+f(z) \phi_{1}{ }^{o}(x, y, t) \\
v(x, y, z, t) & =v^{o}(x, y, t)-z w_{, y}^{o}(x, y, t)+f(z) \phi_{2}{ }^{o}(x, y, t) \\
w(x, y, z, t) & =w^{o}(x, y, t)
\end{aligned}
$$

$$
\text { where } \begin{aligned}
\phi_{1}^{0}(x, y, t) & =\varphi_{1}(x, y, t)+w_{, x}^{0}(x, y, t) ; \\
\phi_{2}^{0}(x, y, t) & =\varphi_{2}(x, y, t)+w_{, y}^{0}(x, y, t) \\
\text { and } \quad f(z) & =z\left[1-(4 / 3)(z / h)^{2}\right] ;
\end{aligned}
$$

Here, the displacement components $u, v$, and $w$ are in directions along the $x-, y$-, and $z$-axes, respectively, at a distance $z$ away from mid plane, and $u^{0}(x, y, t), v^{0}(x, y, t)$ and are displacement components of a generic point on the middle surface. The expressions $\phi_{1}^{0}\left(\varphi_{1}+w_{, x}^{0}\right)$ and $\phi_{2}^{0}\left(\varphi_{2}+w_{, y}^{0}\right)$ represent the total rotations (transverse shear strains) of the normal to the middle surface about the $y$ - and $x$-axes, respectively. The variable $h$ is plate thickness, and ()$_{, x}$ represents the differentiation with respect to $x$. The strain-displacement relations at a distance $z$ away from the mid-plane of a plate can be expressed as:

$$
\begin{gathered}
\epsilon_{x}(x, y, z)=\epsilon_{x}^{o}(x, y, t)-z w_{, x x}^{o}(x, y, t)+f(z) \phi_{1, x}^{o}(x, y, t) \\
\epsilon_{y}(x, y, z, t)=\epsilon_{y}^{o}(x, y, t)-z w_{, y y}^{o}(x, y, t)+f(z) \phi_{2, y}^{o}(x, y, t) \\
\gamma_{x y}(x, y, z, t)=\gamma_{x y}^{o}(x, y, t)-2 z w_{, x y}^{o}(x, y, t)+ \\
f(z) \phi_{1, y}^{o}(x, y, t)+f(z) \phi_{2, x}^{o}(x, y, t) \\
\gamma_{x z}(x, y, z, t)=u_{, z}(x, y, z, t)+w_{, x}(x, y, z, t)= \\
f^{\prime}(z) \phi_{1}^{o}(x, y, t) \\
\gamma_{y z}(x, y, z, t)=v_{, z}(x, y, z, t)+w_{, y}(x, y, z, t)= \\
f^{\prime}(z) \phi_{2}^{o}(x, y, t)
\end{gathered}
$$

Both $\epsilon_{x}^{o}(x, y), \epsilon_{y}^{o}(x, y)$ and $\gamma_{x y}^{o}(x, y)$ are reference surface strains and are defined as:

$$
\begin{aligned}
\epsilon_{y}^{o}(x, y, t) & =v_{, y}^{o}(x, y, t), \quad \epsilon_{x}^{o}(x, y, t)=u_{, x}^{o}(x, y, t), \\
\gamma_{x y}^{o}(x, y, t) & =u_{, y}^{o}(x, y, t)+v_{, x}^{o}(x, y, t)
\end{aligned}
$$

The different components of strain energy in which membrane energy $\left(S^{m}\right)$, bending energy $\left(S^{b}\right)$, additional bending energy due to additional change in curvature $\left(S^{a b}\right)$, and shear energy $\left(S^{S}\right)$ are related respectively to membrane strains $\boldsymbol{\epsilon}^{\mathbf{0}^{\mathbf{T}}}=\left\{\epsilon_{x}^{0}(x, y) \quad \epsilon_{y}^{0}(x, y) \quad \gamma_{x y}^{0}(x, y)\right\}$, bending strains $\boldsymbol{\kappa}^{\boldsymbol{T}}=\left\{-w_{, x x}^{o}(x, y, t)-w_{, y y}^{o}(x, y, t)-2 w_{x y}^{o}(x, y, t)\right\}$, additional bending strains $\boldsymbol{\kappa}^{a^{T}}=$ $\left\{\phi_{1, x}^{o}(x, y, t) \quad \phi_{2, y}^{o}(x, y, t) \quad \phi_{1, y}^{o}(x, y, t)+\phi_{2, x}^{o}(x, y, t)\right\}$, and transverse shear strains $\phi^{\mathbf{0}^{\mathbf{T}}}=$ 
$\left\{\phi_{2}^{0}(x, y, t) \quad \phi_{1}^{0}(x, y, t)\right\}$, through the following constitutive relations:

$$
\begin{gathered}
S^{m}=\frac{1}{2} \int_{A}\left(\epsilon^{\mathbf{0}^{T}} \mathbf{A} \epsilon^{\mathbf{0}}+\epsilon^{\mathbf{0}^{T}} \mathbf{B} \kappa+\epsilon^{\mathbf{0}^{T}} \mathbf{C} \kappa^{a}\right) d A \\
S^{b}=\frac{1}{2} \int_{A}\left(\kappa^{T} \mathbf{B} \epsilon^{0}+\kappa^{T} \mathbf{D} \kappa+\kappa^{T} \mathbf{E} \kappa^{a}\right) d A ; \\
S^{a b}=\frac{1}{2} \int_{A}\left(\kappa^{a^{T}} \mathbf{C} \epsilon^{\mathbf{0}}+\kappa^{a^{T}} \mathbf{E} \kappa+\kappa^{a^{T}} \mathbf{F} \kappa^{a}\right) d A ; \\
S^{s}=\frac{1}{2} \int_{A}\left(\phi^{0^{T}} \mathbf{H} \phi^{0}\right) d A ;
\end{gathered}
$$

The sum of all strain energy of the composite skew plate is calculated as:

$$
S=S^{m}+S^{b}+S^{a b}+S^{s}
$$

In the present investigation, the bold upright letters and bold italic letters are used to denote matrices and vectors, respectively. The extensional stiffness (A), coupling stiffness (B), bending stiffness $(\mathbf{D})$, and shear stiffness $(\mathbf{H})$ matrices are defined in terms of the transformed lamina stiffness $\overline{\mathbf{Q}}$ as:

$$
\begin{aligned}
& \left(A_{i j} B_{i j} D_{i j}\right)=\int_{-h / 2}^{h / 2} \bar{Q}_{i j}\left(1, z, z^{2}\right) d z \\
& =\sum_{k=1}^{l_{n}} \int_{z_{k-1}}^{z_{k}} \bar{Q}_{i j}\left(1, z, z^{2}\right) d z \quad i=1,2,6 \text { and } j=1,2,6 \\
& \left(C_{i j}, E_{i j}, F_{i j}\right)=\int_{-h / 2}^{h / 2} \bar{Q}_{i j}(1, z, f(z)) f(z) d z \\
& =\sum_{k=1}^{l_{n}} \int_{z_{k-1}}^{z_{k}} \bar{Q}_{i j}(1, z, f(z)) f(z) d z i=1,2,6 \text { and } j=1,2,6 \\
& H_{i j}=\int_{-h / 2}^{h / 2} \bar{Q}_{i j} f^{\prime}(z) f^{\prime}(z) d z \\
& =\sum_{k=1}^{l_{n}} \int_{z_{k}}^{z_{k-1}} \bar{Q}_{i j} f^{\prime}(z) f^{\prime}(z) d z \quad i=4,5 \text { and } j=4,5 ;
\end{aligned}
$$

where, $l_{n}$ is the number of orthotropic layer; $A_{i j}, B_{i j}, C_{i j}$, $D_{i j}, E_{i j}, F_{i j}$ and $H_{i j}$ represent plate stiffness; $\bar{Q}_{i j}(i, j=$ $1,2,6)$ are the transformed plane stress reduced stiffness coefficients; and $\bar{Q}_{i j}(i, j=4,5)$ are the transformed throughthickness shear stiffness coefficients.

The potential energy due to in-plane loading can be expressed as:

$$
\begin{aligned}
P=-\frac{1}{2} \int_{0}^{a} \int_{0}^{b} & \left(\left\{\begin{array}{l}
\frac{\partial w^{0}}{\partial x_{0}} \\
\frac{\partial w^{0}}{\partial y}
\end{array}\right\}^{T}\left[\begin{array}{ll}
N x x & N x y \\
N x y & N y y
\end{array}\right]\right. \\
& \left.\left\{\begin{array}{l}
\frac{\partial w^{0}}{\partial x_{0}} \\
\frac{\partial w^{0}}{\partial y}
\end{array}\right\}\right) d x d y
\end{aligned}
$$

where $N_{x x}, N_{y y}$ and $N_{x y}$ are the in-plane loads in the $x$ direction, the in-plane loads in the $y$-direction, and the in-plane shearing loads, respectively. The expression for kinetic energy

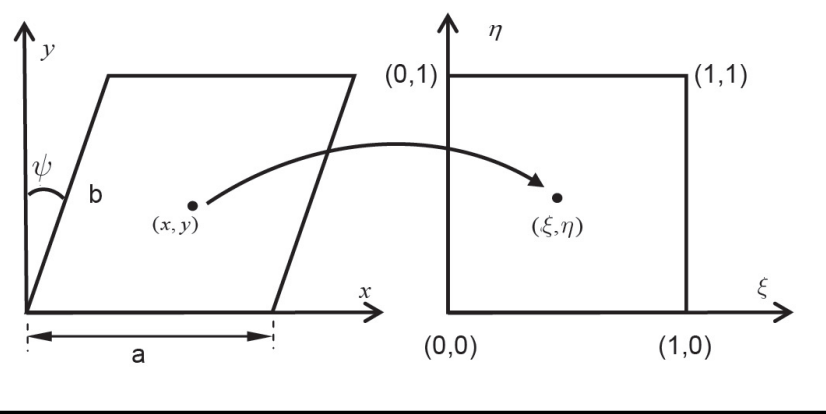

Figure 2. Mapping of the skew plate domain into a unit square plate domain. may be expressed as:

$$
\begin{aligned}
T_{E}=\frac{1}{2} \int_{0}^{a} \int_{0}^{b} & \left\{\begin{array}{l}
\frac{\partial u^{0}}{\partial t} \\
\frac{\partial v^{0}}{\partial t^{0}} \\
\frac{\partial w^{0}}{\partial \phi^{0}} \\
\frac{\partial \phi_{2}}{\partial t} \\
\frac{\partial \phi_{1}^{0}}{\partial t}
\end{array}\right\}^{T}\left[\begin{array}{ccccc}
\rho h & 0 & 0 & 0 & 0 \\
0 & \rho h & 0 & 0 & 0 \\
0 & 0 & \rho h & 0 & 0 \\
0 & 0 & 0 & \frac{\rho h^{3}}{12} & 0 \\
0 & 0 & 0 & 0 & \frac{\rho h^{3}}{12}
\end{array}\right] \\
& \left.\left\{\begin{array}{c}
\frac{\partial u^{0}}{\partial t} \\
\frac{\partial v^{0}}{\partial t} \\
\frac{\partial w^{0}}{\partial t} \\
\frac{\partial \phi_{0}^{0}}{\partial t} \\
\frac{\partial \phi_{1}^{0}}{\partial t}
\end{array}\right\}\right) d x d y ;
\end{aligned}
$$

where $\rho$ is the density. The energy expression $S^{m}, S^{b}, S^{a b}, S^{s}$ and $P$ is transformed from the orthogonal co-ordinate system to the oblique co-ordinate system using transformation relations. Mapping of a skew plate having length " $a$," breadth " $b$," and thickness " $h$," into a unit square plate domain in the $\xi-\eta$ plane is shown in Fig. 2. The transformation relations are given by:

$$
x=a \xi+b \eta \sin \psi ; \quad y=b \eta \cos \psi ;
$$

The following transformation relations are followed for the mapping and function of the functional from the $x-y$ plane to the $\xi-\eta$ plane:

$$
\begin{aligned}
{\left[\begin{array}{c}
\frac{\partial}{\partial x} \\
\frac{\partial}{\partial y}
\end{array}\right] } & =\left[\begin{array}{cc}
\frac{1}{a} & 0 \\
-\frac{\tan \psi}{a} & \frac{\sec \psi}{b}
\end{array}\right]\left[\begin{array}{c}
\frac{\partial}{\partial \xi} \\
\frac{\partial}{\partial \eta}
\end{array}\right] ; \\
{\left[\begin{array}{c}
\frac{\partial^{2}}{\partial x^{2}} \\
\frac{\partial^{2}}{\partial y^{2}} \\
\frac{\partial^{2}}{\partial x \partial y}
\end{array}\right]=} & {\left[\begin{array}{ccc}
\frac{1}{a^{2}} & 0 & 0 \\
\frac{\tan ^{2} \psi}{a^{2}} & \frac{\sec ^{2} \psi}{b^{2}} & -\frac{2 \tan \psi \sec \psi}{a b} \\
-\frac{\tan \psi}{a^{2}} & 0 & \frac{\sec \psi}{a b}
\end{array}\right] } \\
& {\left[\begin{array}{c}
\frac{\partial^{2}}{\partial \xi^{2}} \\
\frac{\partial^{2}}{\partial \eta^{2}} \\
\frac{\partial^{2}}{\partial \xi \partial \eta}
\end{array}\right] ; }
\end{aligned}
$$

The transformed membrane strain energy $S^{* m}, S^{* b}, S^{* a b}$ and $S^{* s}$ potential energy $\left(P^{*}\right)$ are kinetic energy $\left(T_{E}^{*}\right)$ represented as:

$$
\begin{aligned}
S^{* m}= & \frac{1}{2} \int_{0}^{1} \int_{0}^{1} \Re_{1}\left(u_{, \xi}^{o}, u_{, \eta}^{o}, v_{, \xi}^{o}, v_{, \eta}^{o}\right) \\
& \cos \psi d \xi d \eta ;
\end{aligned}
$$




$$
\begin{aligned}
\hline S^{* b}= & \frac{1}{2} \int_{0}^{1} \int_{0}^{1} \Re_{2}\left(w_{, \xi \xi}^{o}, w_{, \xi \eta}^{o}, w_{, \eta \eta}^{o}, \phi_{1, \xi}^{o}, \phi_{1, \eta}^{o}, \phi_{2, \xi}^{o}, \phi_{2, \eta}^{o}\right) \\
& \cos \psi d \xi d \eta ; \\
S^{* a b}= & \frac{1}{2} \int_{0}^{1} \int_{0}^{1} \Re_{3}\left(w_{, \xi \xi}^{o}, w_{, \xi \eta}^{o}, w_{, \eta \eta}^{o}, \phi_{1, \xi}^{o}, \phi_{1, \eta}^{o}, \phi_{2, \xi}^{o}, \phi_{2, \eta}^{o}\right)
\end{aligned}
$$

$\cos \psi d \xi d \eta$

$$
\begin{gathered}
S^{* s}=\frac{1}{2} \int_{0}^{1} \int_{0}^{1} \Re_{4}\left(\phi_{1}^{o}, \phi_{2}^{o}\right) \cos \psi d \xi d \eta \\
P^{*}=-\frac{1}{2} \int_{0}^{1} \int_{0}^{1} \Re_{5}\left(n_{x x}, n_{x y}, n_{y y}, w_{, \xi}^{o}, w_{, \eta}^{o}\right) \\
\cos \psi d \xi d \eta ; \\
T_{E}^{*}=\frac{1}{2} \int_{0}^{1} \int_{0}^{1} \Re_{6}\left(u^{o}, v^{o}, w^{o}, \phi_{1}^{o}, \phi_{2}^{o}\right) \cos \psi d \xi d \eta
\end{gathered}
$$

The expressions for $\left.\Re_{i} i=1,2, \ldots, 6\right)$ are given in Appendix A.

\subsection{Generation of Orthogonal Polynomials}

To generate orthogonal polynomials satisfying essential boundary conditions over the unit square plate domain in the $\xi-\eta$ plane, the following displacement fields are used:

$$
\begin{aligned}
\tilde{u}^{o}(\xi, \eta, t) & =\sum_{j=1}^{N^{*}} U_{j} \hat{\alpha}_{j}(\xi, \eta) \cos (\omega t) \\
\tilde{v}^{o}(\xi, \eta, t) & =\sum_{j=1}^{N^{*}} V_{j} \hat{\beta}_{j}(\xi, \eta) \cos (\omega t) \\
\tilde{w}^{o}(\xi, \eta, t) & =\sum_{j=1}^{N^{*}} W_{j} \hat{\Theta}_{j}(\xi, \eta) \cos (\omega t) \\
\tilde{\phi}_{1}{ }^{o}(\xi, \eta, t) & =\sum_{j=1}^{N^{*}} K_{j} \hat{\gamma}_{j}(\xi, \eta) \cos (\omega t) \\
\tilde{\phi}_{2}{ }^{o}(\xi, \eta, t) & =\sum_{j=1}^{N^{*}} L_{j} \hat{\delta}_{j}(\xi, \eta) \cos (\omega t)
\end{aligned}
$$

where $N^{*}$ is the order of approximation to get the desired accuracy; $U_{j}, V_{j}, W_{j}, K_{j}$ and $L_{j}$ are unknown constants; and $\hat{\alpha}_{j}(\xi, \eta), \hat{\beta}_{j}(\xi, \eta), \hat{\Theta}_{j}(\xi, \eta), \hat{\gamma}_{j}(\xi, \eta)$ and $\hat{\delta}_{j}(\xi, \eta)$ are boundary characteristics orthonormal polynomial (BCOP) functions.

The orthonormal polynomials $\left(\hat{\Theta}_{j}(\xi, \eta)\right.$ are generated for satisfying the essential boundary conditions over the unit square plate domain in the $\xi, \eta$ plane by the Gram-Schmidt process explained by Singh and Chakraverty. ${ }^{4}$ In the present study, the same orthonormal polynomials are considered for $\hat{\alpha}_{j}(\xi, \eta), \hat{\beta}_{j}(\xi, \eta), \hat{\gamma}_{j}(\xi, \eta)$ and $\hat{\delta}_{j}(\xi, \eta)$ also. Orthonormal polynomials $\left(\hat{\Theta}_{j}(\xi, \eta)\right)$ over the region $0 \leq \xi \leq 1,0 \leq \eta \leq 1$ have been generated using the linearly independent set of functions $F_{j}(\xi, \eta)=f(\xi, \eta) \times f_{j}(\xi, \eta)$. Both $f(\xi, \eta)$ and $f_{j}(\xi, \eta)$ are two functional scalar quantities as given below:

$$
f(\xi, \eta)=\xi^{p}(1-\xi)^{q} \eta^{r}(1-\eta)^{s} ;
$$

$$
f_{j}(\xi, \eta)=\left\{1, \xi, \eta, \xi^{2}, \xi \eta, \eta^{2}, \xi^{3}, \xi^{2} \eta, \xi \eta^{2}, \eta^{3}, \ldots\right\} .
$$

The explicit expression for the orthogonal polynomials for different types of boundary conditions are derived by using the following equations:

$$
\Theta_{j}(\xi, \eta)=\xi^{p} \eta^{q}(1-\xi)^{r}(1-\eta)^{s} f_{j}(\xi, \eta)
$$

The value of ' $p$ ' depends on the boundary condition on $\xi=$ 0,1 . At $\xi=0, p=0,1,2$ respectively for free, simply supported, and clamped boundary conditions. At $\xi=1, q=$ $0,1,2$ respectively for free, simply supported, and clamped boundary conditions. In a similar way, the values of $r$ and $s$ are chosen for $\eta=0,1$ as:

$\Theta_{1}(\xi, \eta)=F_{1}(\xi, \eta), \Theta_{j}(\xi, \eta)=F_{j}(\xi, \eta)-\sum_{i=1}^{j-1} \alpha_{j i} \Theta_{i}(\xi, \eta)$

$$
\begin{aligned}
\alpha_{j i} & =\frac{<F_{j}(\xi, \eta), \Theta_{i}(\xi, \eta)>}{<\Theta_{i}(\xi, \eta), \Theta_{i}(\xi, \eta)>} \\
i & =1,2,3, \ldots,(j-1), \quad j=2,3,4, \ldots, N^{*} .
\end{aligned}
$$

The inner product of the functions $\Theta_{i}(\xi, \eta)$ and $\Theta_{j}(\xi, \eta)$ is expressed as:

$$
<\Theta_{i}(\xi, \eta), \Theta_{j}(\xi, \eta)>=\int_{0}^{1} \int_{0}^{1} \Theta_{i}(\xi, \eta) \Theta_{j}(\xi, \eta) d \xi d \eta
$$

The norm of the function $\Theta_{j}(\xi, \eta)$ is expressed by:

$$
\begin{aligned}
\left\|\Theta_{j}(\xi, \eta)\right\| & =<\Theta_{j}(\xi, \eta), \Theta_{j}(\xi, \eta)>^{1 / 2} \\
& =\left[\int_{0}^{1} \int_{0}^{1} \Theta_{j}(\xi, \eta) \Theta_{j}(\xi, \eta) d \xi d \eta\right]^{1 / 2} .
\end{aligned}
$$

The normalisation of the orthogonal polynomial is essential for avoiding the illness of the stiffness matrix. The normalisation is done by:

$$
\hat{\Theta}_{j}(\xi, \eta)=\frac{\Theta_{j}(\xi, \eta)}{\left\|\Theta_{j}(\xi, \eta)\right\|} .
$$

In the present study for all cases of the skew plate, 36 numbers of terms have been considered for getting the converged pre-buckling free vibration frequency and buckling load. The explicit expressions for 36 polynomial terms for simply supported boundary conditions (SSSS) are given in Appendix B. For the sake of brevity, the explicit expressions for polynomial terms for other boundary conditions are not presented.

\subsection{Rayleigh-Ritz Method}

The total potential energy $(\Pi)$ of the system is the sum of the strain energy $(S)$ and the potential energy $(P)$. The total potential energy function $\Pi(S+P)$ and kinetic energy function $\left(T_{E}\right)$ are transformed from the $x-y$ plane to the $\xi-\eta$ plane by using Eqs. (14) and (15). The displacement and rotational components $\left(\tilde{u}^{o}(\xi, \eta, t), \tilde{v}^{o}(\xi, \eta, t), \tilde{w}^{o}(\xi, \eta, t), \tilde{\phi}_{1}^{o}(\xi, \eta, t)\right.$ and $\left.\tilde{\phi}_{2}^{o}(\xi, \eta, t)\right)$ are expressed in terms of unknown constants $\left(U_{j}\right.$, $\left.V_{j}, W_{j}, K_{j}, L_{j}\right)$ using the orthonormal polynomials $\left(\hat{\alpha}_{j}(\xi, \eta), \hat{\beta}_{j}(\xi, \eta), \hat{\Theta}_{j}(\xi, \eta), \hat{\gamma}_{j}(\xi, \eta)\right.$ and $\left.\hat{\delta}_{j}(\xi, \eta)\right)$. Further, these displacement and rotational components are substituted 
in the total potential energy function and the kinetic energy function to get the energy function in the $\xi-\eta$ plane. The total energy $\left(T_{L}\right)$ is the addition of the kinetic energy $\left(T_{E}^{*}\right)$ to the total potential energy ( $\Pi)$. This involves different unknowns, $U_{j}, V_{j}, W_{j}, K_{j}$ and $L_{j}$, and can be minimised with respect to constants as follows:

$$
\frac{\partial T_{L}}{\partial C_{j}}=0 \quad \text { for } \quad j=1,2,3, \ldots, 5 N^{*}
$$

where,

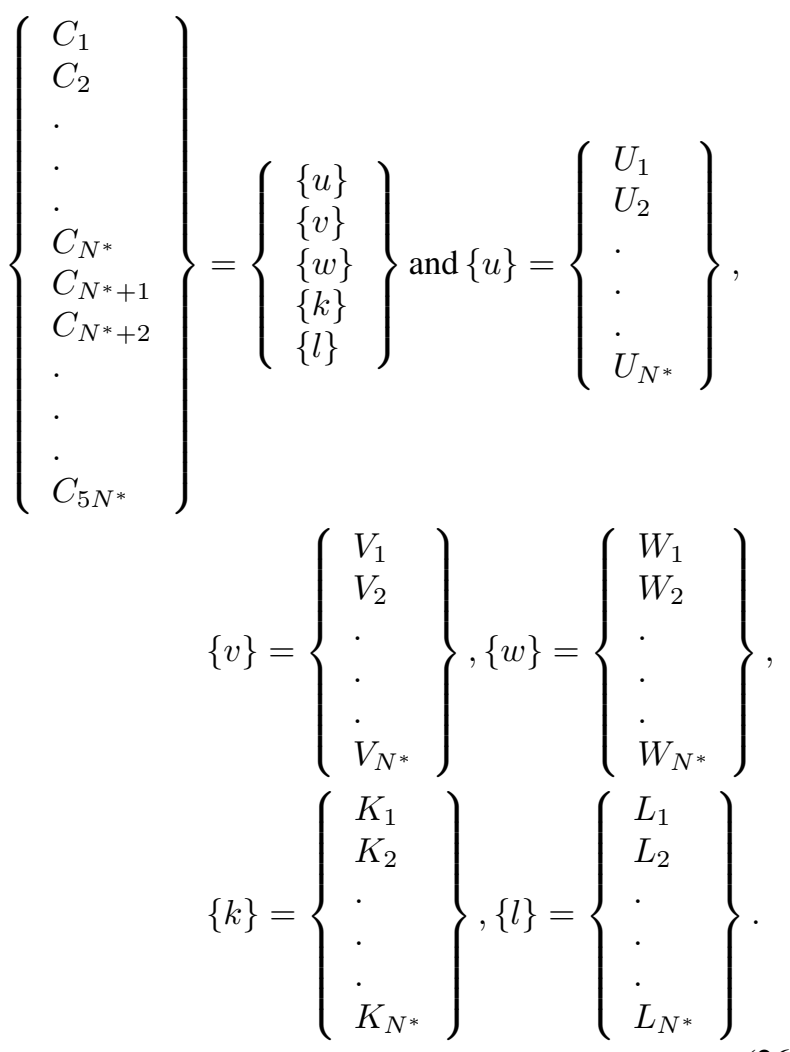

The total number of constants is $5 N^{*}$. The above Eq. (26a) leads to the governing eigenvalue equation for finding the prebuckling free vibration frequency $\left(\lambda_{f}\right)$ as given below:

$$
\sum\left[\left[K_{L}-\lambda K_{G}\right]-\lambda_{f} M\right]\left\{\begin{array}{c}
\{u\} \\
\{v\} \\
\{w\} \\
\{k\} \\
\{l\}
\end{array}\right\}=\{0\} ;
$$

where $[\mathrm{M}]$ is the mass matrix; $\left[K_{L}\right]$ is the linear elastic stiffness; and $\left[K_{G}\right]$ is the linear geometric stiffness matrix. For finding the buckling load $\left(\lambda_{b u c k}\right)$ in the static buckling problem, the eigenvalue problem is reduced to the following:

$$
\sum\left[K_{L}-\lambda_{b u c k} K_{G}\right]\left\{\begin{array}{c}
\{u\} \\
\{v\} \\
\{w\} \\
\{k\} \\
\{l\}
\end{array}\right\}=\{0\} .
$$

Here, $\lambda<\lambda_{\text {buck }}$. Equations (26) and (27) constitutes a set of $5 N^{*}$ simultaneous algebraic equations referred to as the Ritz system.

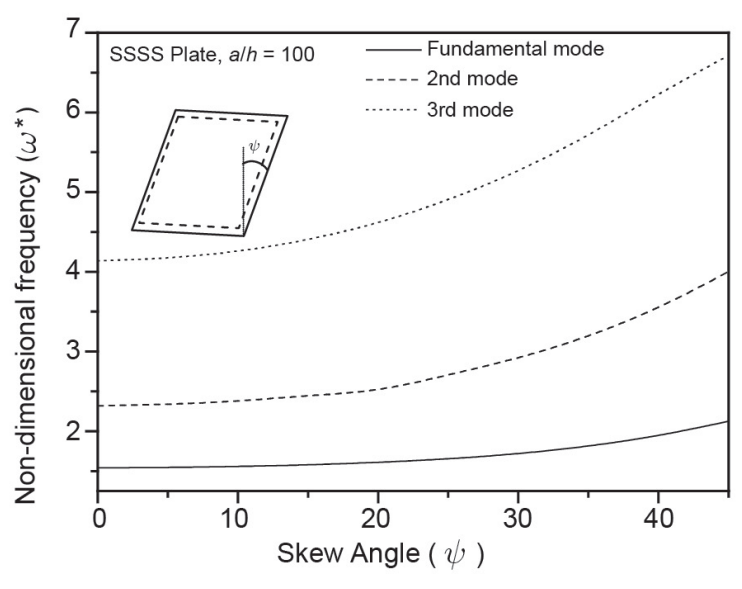

Figure 3. Variation of non-dimensional frequency $\left(\omega^{*}\right)$ for different modes of vibration for three layered crosspl- [0/90/0] composite SSSS skew plates $(a / h=100)$ having different skew angles.

\section{RESULTS AND DISCUSSIONS}

The non-dimensional fundamental frequency $\left(\omega^{*}\right)$ of isotropic skew plates $(a / b=1, a / h=100)$ for different skew angles $(\psi)$ and different support conditions are similar to open literature and given in Table 1. For obtaining the convergence of results, 36 numbers of terms (orthogonal polynomials) are considered for all cases of support conditions and skew angles. The present solution agrees well with the results obtained by using the double series of beam characteristic function by Nair and Durvasula and the two-dimensional orthogonal plate functions by Liew and Lam. ${ }^{2,31}$ Durvasula used the double Fourier sine series to find the non-dimensional fundamental frequency of a simply supported skew plate. ${ }^{1}$

The influence of skew angles on the free transverse vibration of simply supported composite [0/90/0] skew plates is shown in Fig. 3. As the skew angle increases $0 \leq \psi \leq 45^{0}$, the frequency of the first three modes of vibration increases. This behaviour is expected sincebecause, when the skew angle increases, the stiffness of the plate increases, which makes a higher frequency for all three modes of vibration. Similar behaviour is also observed for skew plates with more and more edge restraints. Figure 4 a shows the effect of boundary conditions on the fundamental frequency of a composite skew plate. As the four edges of the composite [0/90/0] skew plate support changes in boundary conditions from simply supported (S) to clamped (C) one after another, the stiffness increases, which causes a higher frequency value. The fundamental frequencies of the composite [0/90/0] skew plate are shown for the following four boundary conditions: SSSS, CSSS, CSCS and CCCC. The pre-buckling vibration behaviour is presented as a plot of non-dimensional fundamental frequencies against dimensionless in-plane loads for composite skew plates of different boundary conditions as shown in Fig. 4b. The pre-buckling fundamental frequency and in-plane load of each plate are normalized with respect to the free vibration frequency and buckling load of an SSSS plate $\left(a / b=1 ; \psi=30^{\circ} ; a / h=100\right)$. The result indicates that the fundamental pre-buckling frequency increases with the increase in the edge restraints of the skew plate. The frequency is always higher for a CSCS plate as compared to an SCSC plate. 
Table 1. Non-dimensional fundamental frequency $\left(\omega^{*}\right)$ of isotropic square skew plates $(a / b=1, a / h=100)$ for different skew angles $(p s i)$ and different support conditions.

\begin{tabular}{|c|c|c|c|c|}
\hline \multirow{2}{*}{ Type of Supports } & \multirow{2}{*}{ Skew angle $(\psi)$} & \multicolumn{3}{|c|}{ Non-dimensional fundamental frequency $\left(\omega^{*}\right)$} \\
\hline & & Present solution & Durvasula $^{1}$ & Liew and $\mathrm{Lam}^{2}$ \\
\hline \multirow{4}{*}{ SSSS } & $0^{\circ}$ & 1.99 & 2.0 & - \\
\hline & $15^{\circ}$ & 2.11 & 2.11 & 2.11 \\
\hline & $30^{\circ}$ & 2.52 & 2.54 & 2.52 \\
\hline & $45^{\circ}$ & 3.53 & 3.54 & 3.53 \\
\hline & & & Nair and Durvasula ${ }^{31}$ & Liew and $\mathrm{Lam}^{2}$ \\
\hline \multirow{4}{*}{ CSSS } & $0^{\circ}$ & 2.39 & 2.40 & 2.40 \\
\hline & $15^{\circ}$ & 2.54 & 2.54 & 2.55 \\
\hline & $30^{\circ}$ & 3.10 & 3.11 & 3.12 \\
\hline & $45^{\circ}$ & 4.53 & 4.53 & 4.58 \\
\hline \multirow{4}{*}{ CCSS } & $0^{\circ}$ & 2.74 & 2.74 & 2.74 \\
\hline & $15^{\circ}$ & 2.90 & 2.91 & 2.91 \\
\hline & $30^{\circ}$ & 3.52 & 3.57 & 3.52 \\
\hline & $45^{\circ}$ & 5.08 & 5.12 & 5.08 \\
\hline
\end{tabular}

Note: $\omega^{*}=\omega\left[\left(b^{2} / \pi^{2}\right) \sqrt{\rho h / D}\right] ; D$ is flexural rigidity.

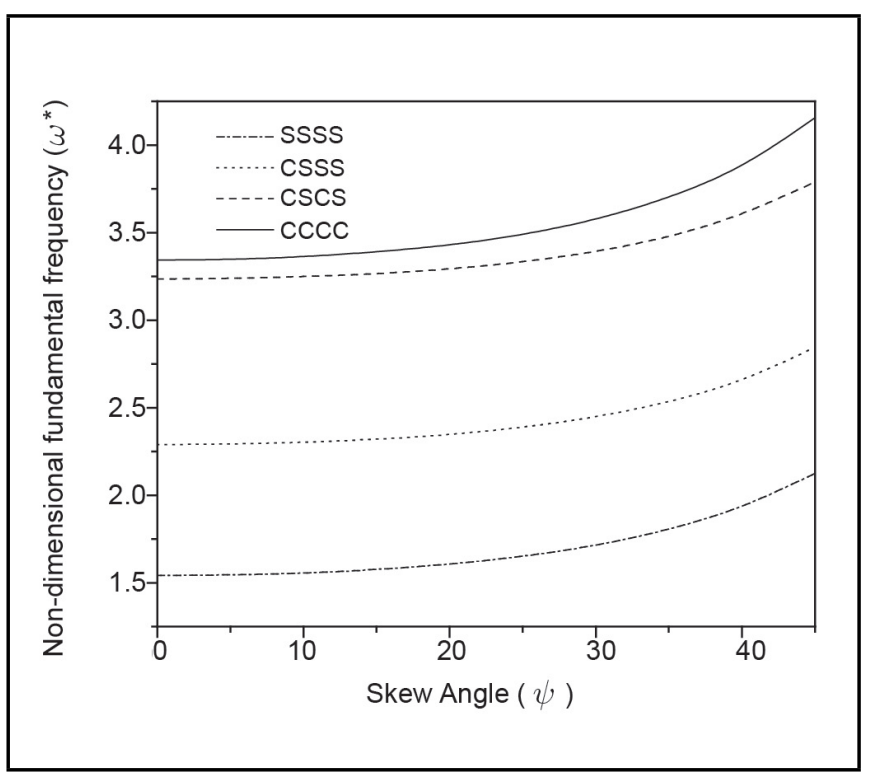

Figure 4a. Variation of non-dimensional fundamental frequency $\left(\omega^{*}\right)$ for different boundary conditions for three layered cross-ply [0/90/0] composite skew plates $(a / h=100)$ having different skew angles.

Figure 5a shows the influence of aspect ratio on the free vibration frequency of skew plates. As the aspect ratio $(a / b)$ increases, the non-dimensional fundamental frequency increases. This is because the plate becomes stiffer with the increase of the aspect ratio. It is also observed that as the aspect ratio increases, the non-dimensional fundamental frequency increases more rapidly for plates having a higher skew angle. Figure $5 \mathrm{~b}$ shows the effect of the aspect ratio on the second mode of transverse vibration of skew plates. The variation of second mode frequency is similar to the fundamental frequency for a plate with an aspect ratio up to nearly 1.5 for all values of skew angles. However, the frequency variation decreases beyond an aspect ratio of 1.5 for all values of skew angles. The pre-buckling vibration behaviour is presented as a plot of non-dimensional fundamental frequencies against dimensionless in-plane loads for SSSS composite skew plates of different skew angles as shown in Fig. 5c. The pre-buckling fundamental frequency and in-plane load of each plate are normalized with respect to the free vibration frequency and buckling load of an SSSS plate $\left(a / b=1 ; \psi=0^{\circ} ; a / h=100\right)$. The result indicates that the stiffness of the skew plate increases

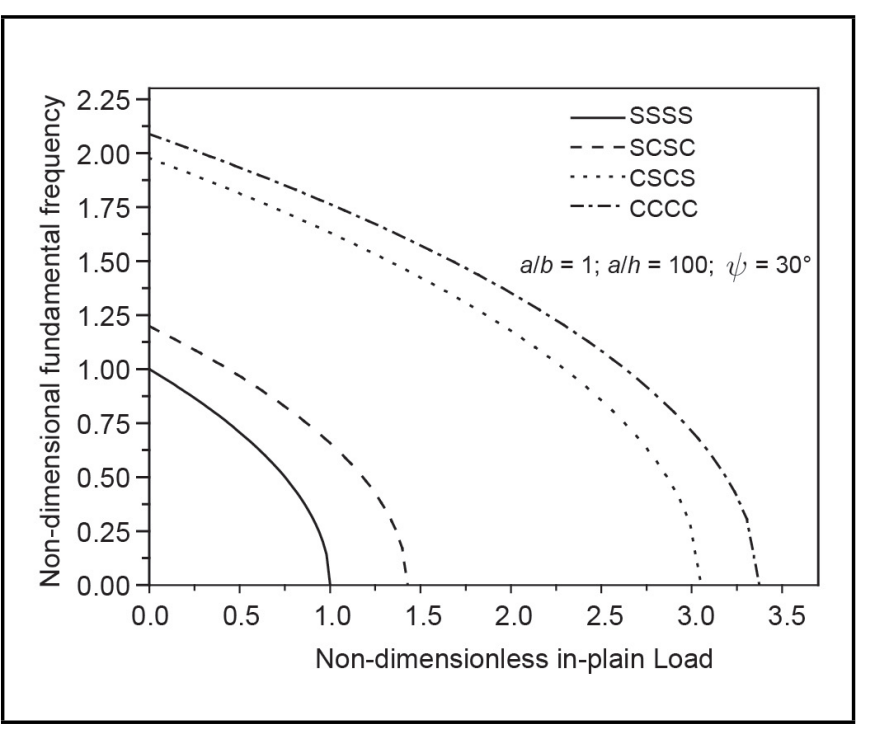

Figure 4b. The influence of skew angle on the pre-buckling vibration behaviour of a composite skew plate $a / b=1 ; a / h=100 ; \psi=300$ ) having different boundary conditions.

in a non-uniform manner with the increase of the skew angle. Also, in the pre-buckling range, frequency decreases with the increase of in-plane load, reaching zero frequency at buckling load.

Figure 6 reflects on the effect of shear deformation on transverse vibration of skew plates for different aspect ratios $(a / b)$ of the three layered cross-ply [0/90/0] composite SSSS skew plates $\psi=30^{\circ}$. The effect of shear deformation on frequency is more for higher values of the aspect ratio. The frequency of different modes of vibration increases with the increase of aspect ratio until a certain value, and after that, it remains constant or slowly decreases for a plate of higher thickness in which $a / h=10$ and 20. The non-dimensional fundamental frequencies increase with the increase of aspect ratio $(a / b)$ for plates of $a / h$ ratio 50 and 100 . For plates of $a / h$ ratio 10 and 20 , the non-dimensional fundamental frequency increases up to an aspect ratio $(a / b)$ with values of 1.75 and 2.5 , respectively, and then the frequency value slowly decreases.

Figure 7 shows the influence of different types of linearly varying in-plane loading on the pre-buckling vibration behaviour of an SSSS composite plate $\left(a / b=1 ; \psi=30^{\circ}\right.$; $a / h=100)$. The pre-buckling fundamental frequency and 


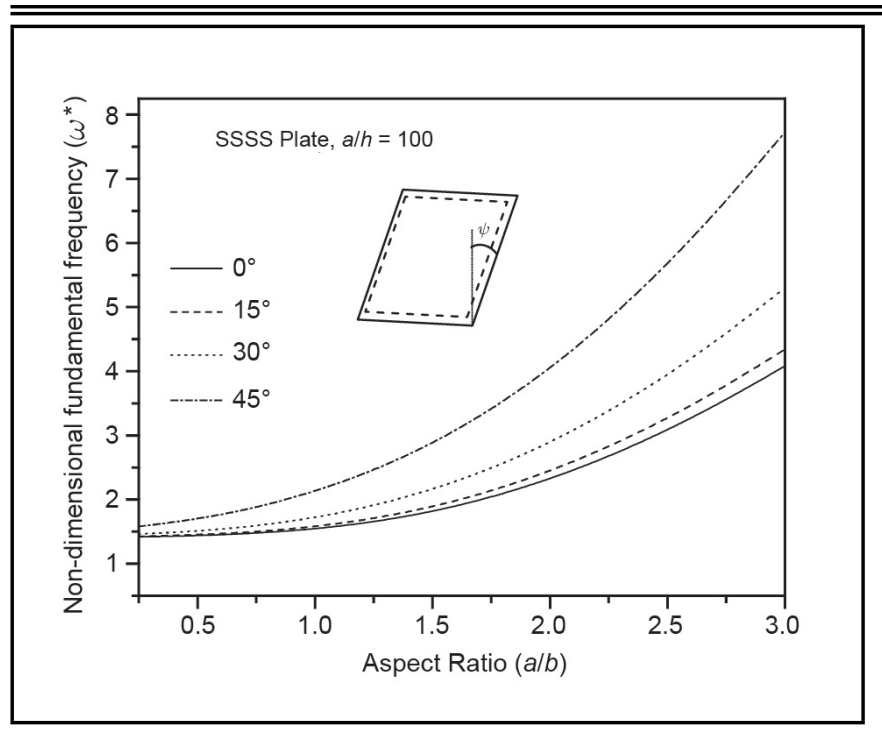

Figure 5a. Variation of non-dimensional fundamental frequency $\left(\omega^{*}\right)$ with respect to the aspect ratio $(a / b)$ for three layered cross-ply [0/90/0] composite SSSS skew plates $(a / h=100)$ having different skew angles.

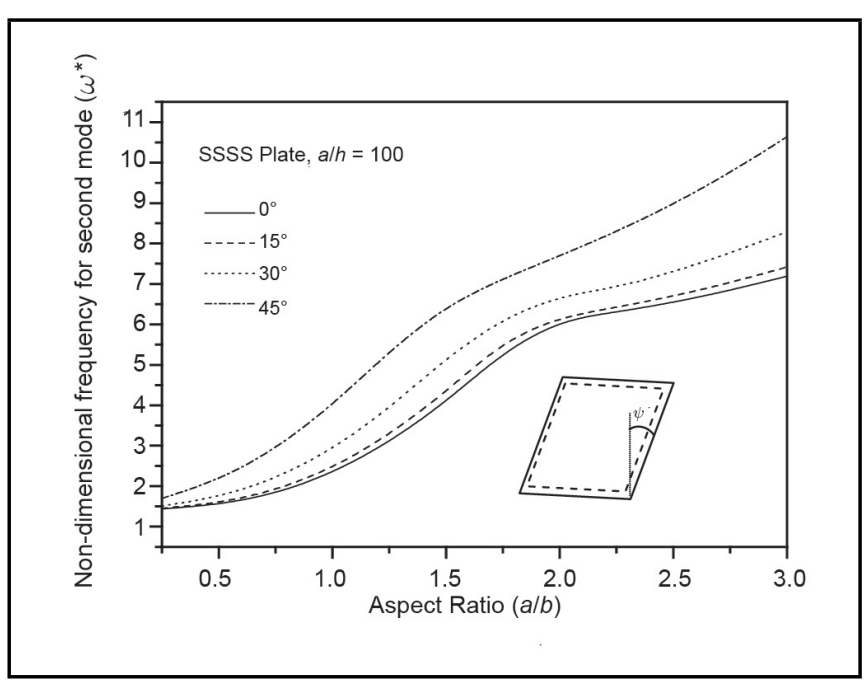

Figure 5b. Variation of non-dimensional frequency $\left(\omega^{*}\right)$ for the second mode with respect to the aspect ratio $(a / b)$ for three layered cross-ply [0/90/0] composite SSSS skew plates $(a / h=100)$ having different skew angles.

in-plane load of each plate are normalized with respect to the free vibration frequency and buckling load of the SSSS plate $\left(a / b=1 ; \psi=30^{\circ} ; a / h=100\right)$. The result indicates that the pre-buckling fundamental frequency value increases with the increase in the $\gamma$ value. Also, in the pre-buckling range, frequency decreases with the increase of in-plane load, reaching zero frequency at buckling load. The buckling loads obtained from the present method of solution for skew plates subjected to uniform in-plane loading with boundary conditions in which all edges are simply supported and in which all edges are clamped are similar to open literature and given in Table 2. The mechanical properties used in the present analysis for composite skew plate are: $E_{11} / E_{22}=25, G_{12}=G_{13}=$ $0.5 E_{22}, G_{23}=0.2 E_{22}$ and $\nu_{12}=0.25$. The variation of dimensionless buckling load coefficients $\mathrm{k}_{c}\left(=\frac{N_{c r} b^{2}}{E_{22} \pi^{2} h^{3}}\right)$ for different plate aspect ratios $(a / b)$ of a simply supported cross-ply composite [0/90/0] skew plate $\left(a / h=100, \psi=30^{c} i r c\right)$ subjected to different types of in-plane loading $(\gamma=0.5,1.0,1.5$ and 2.0) are shown in Fig. 8. It is observed that for uniform in-plane loading $(\gamma=0)$, the value of the buckling coefficient

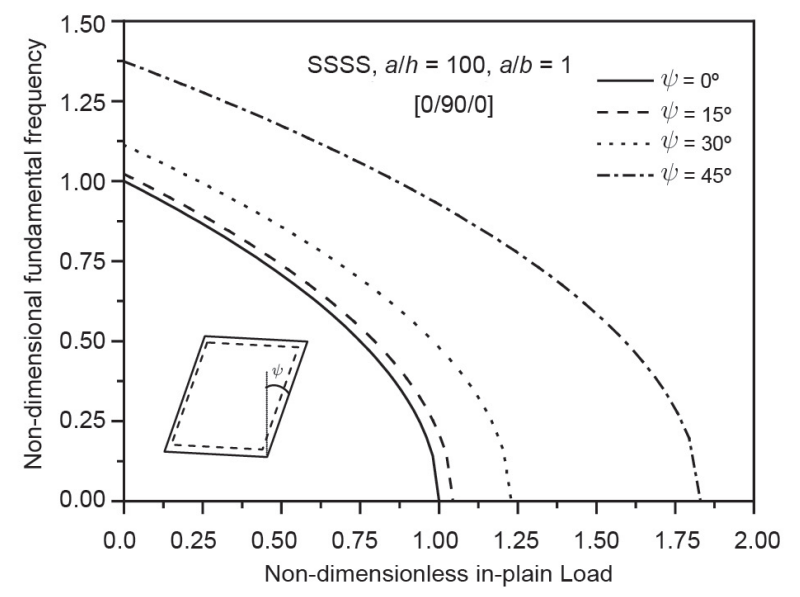

Figure 5c. The influence of skew angle on the pre-buckled vibration behaviour of an SSSS composite skew plate $(a / b=1 ; a / h=100)$.

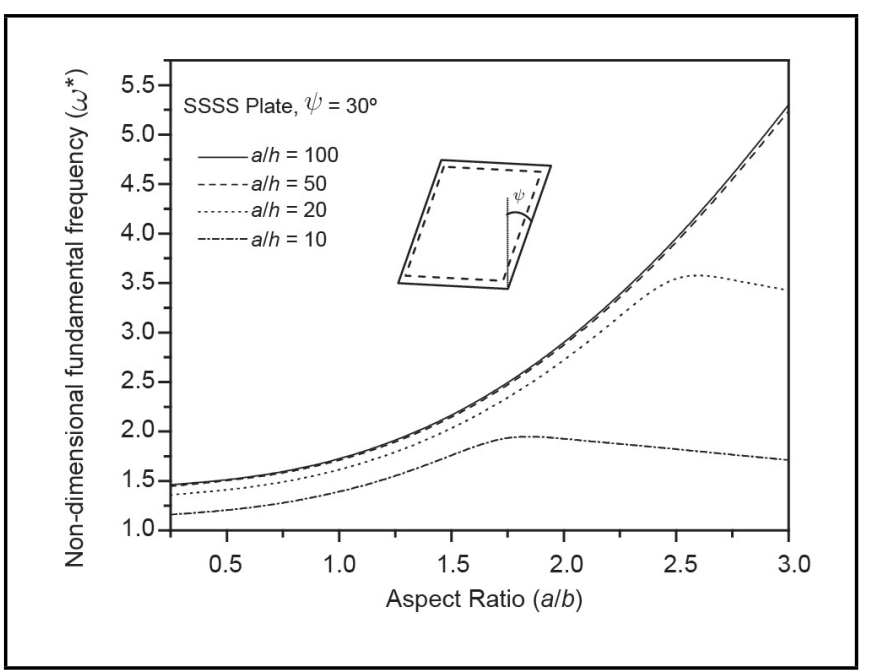

Figure 6. Effect of shear deformation on non-dimensional fundamental frequency $\left(\omega^{*}\right)$ for different aspect ratios $(a / b)$ of the three layered cross-ply [0/90/0] composite SSSS skew plates $(\psi=300)$.

(k) for the composite skew plate decreases up to an aspect ratio $(a / b)$ of 1.75 and then slowly increases up to an aspect ratio value of 2.35 and then decreases slowly. Here, the aspect ratio at which the composite skew plate buckling mode shifts from first mode to second mode is 2.35. For other types of linearly varying in-plane loading when $\gamma=0.5,1.0,1.5$ and 2.0 the value of the buckling coefficient first decreases up to an aspect ratio $(a / b)$ of $1.45,1.10,0.9$ and 0.8 , respectively. The mode-shifting occurs from first mode to second mode for different types of linearly varying in-plane loading in which $\gamma=0.5,1.0,1.5$ and 2.0 for aspect ratios $(a / b)$ of 2.25, 2.0, 1.33 , and 1.0 , respectively. For all types of linearly varying inplane loading, the value of the buckling coefficient decreases after mode-shifting from first mode to second mode.

Figure 9 shows the variation of the dimensionless buckling load coefficient $\mathrm{k}_{c}\left(=\frac{\mathrm{N}_{c r} \mathrm{~b}^{2}}{\mathrm{E}_{22} \pi^{2} \mathrm{~h}^{3}}\right)$ for different plate aspect ratios $(a / b)$ of a clamped cross-ply composite [0/90/0] skew plate $(a / h=100)$ subjected to in-plane bending loading $(\gamma=2$.). For in-plane bending, the value of the buckling coefficient first decreases up to an aspect ratio of nearly 0.8 and then increases up to an aspect ratio of nearly 1.0 for a skew plate having a 
Table 2. Critical buckling coefficient $\left(k_{i}\right)$ isotropic square skew plates $(a / b=1, a / h=100)$ for different skew angles $(\psi)$ under uniform in-plane loading.

\begin{tabular}{|c|c|c|c|c|}
\hline \multirow{2}{*}{ Type of Supports } & \multirow{2}{*}{ Skew angle $(\psi)$} & \multicolumn{3}{|c|}{ Dimensionless buckling coefficient $\left(k_{i}\right)$} \\
\hline & & Present solution & Wang $^{5}$ & Babu and Kant ${ }^{32}$ \\
\hline \multirow{4}{*}{ Simply supported plate (SSSS) } & $0^{\circ}$ & 4 & 4.0 & 4.0 \\
\hline & $15^{\circ}$ & 3.826 & 3.824 & 3.830 \\
\hline & $30^{\circ}$ & 3.323 & 3.316 & 3.330 \\
\hline & $45^{\circ}$ & 2.559 & 2.525 & 2.557 \\
\hline & & & Durvasula $^{33}$ & Wang et $\mathrm{al}^{3}$ \\
\hline \multirow{4}{*}{ Clamped supported plate (CCCC) } & $0^{\circ}$ & 10.074 & 10.074 & 10.074 \\
\hline & $15^{\circ}$ & 9.431 & 9.462 & 9.479 \\
\hline & $30^{\circ}$ & 7.612 & 7.638 & 7.734 \\
\hline & $45^{\circ}$ & 5.110 & 5.110 & 5.172 \\
\hline
\end{tabular}

Note: $k_{i}=N_{c r} b^{2} \cos ^{4} \psi / \pi^{2} D$

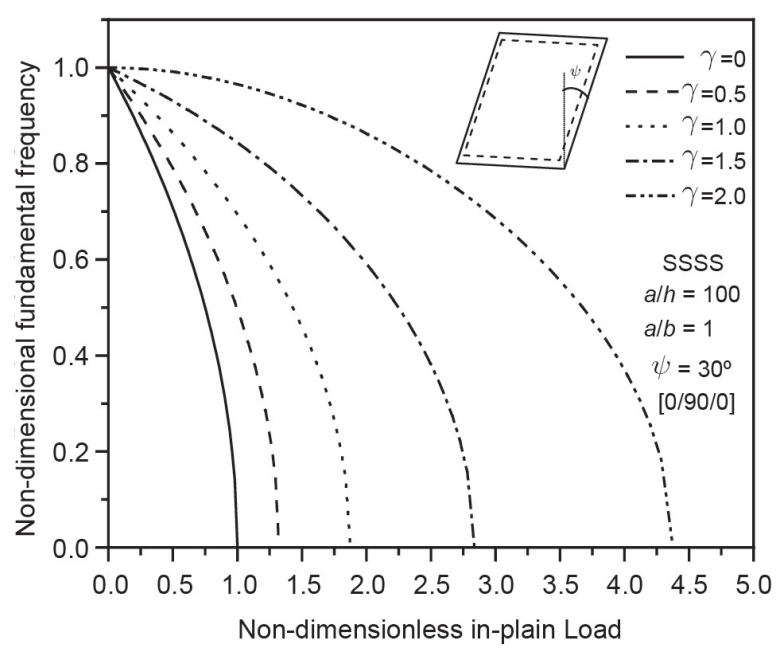

Figure 7. The influence of different types of in-plane loading on the prebuckled vibration behaviour of cboxan SSSS composite skew plate $(a / b=1$; $a / h=100 ; \psi=30^{\circ}$ ).

skew angle of $\psi=30^{\circ}$ and $45^{\circ}$. For the skew angle of $\psi=30^{\circ}$ and $15^{\circ}$, the buckling coefficient value mostly remains constant between aspect ratio values of 0.8 and 1.0. The change of mode occurs for all skew plates at an aspect ratio of 1.0, and the further the buckling coefficient value decreases, the higher the value of the aspect ratio.

In the present investigation, the following nine sets of boundary conditions are considered: SSSS, SSCS, SCSS, CSCS, SCSC, SSCC, CCSC, CCCS, CCCC, where 'S" stands for simply supported edge and 'C' for clamped edge. The letters indicate the boundary conditions on the edge of the plate in the anti-clockwise fashion starting from the left-hand corner. Table 3 shows the dimensionless buckling coefficient of three layered cross-ply [0/90/0] composite skew plates $\left(\psi=45^{\circ}\right.$, $a / h=100)$ with nine sets of boundary conditions subjected to trapezoidal in-plane loading $(\gamma=0.5)$. It is observed that the more edge restraint there is, the higher the buckling coefficient is.

Tables 4 and 5 show the dimensionless buckling coefficient for three layered cross-ply [0/90/0] composite skew plates $\left(\psi=45^{\circ}, a / h=100\right)$ with nine sets of boundary conditions subjected to triangular in-plane loading and partial tension inplane loading, respectively.

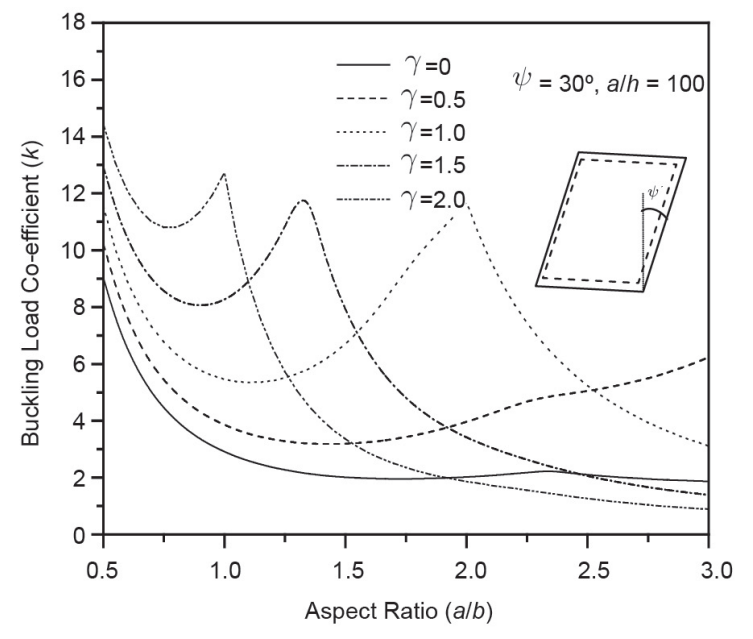

Figure 8. Variation of the buckling load coefficient $(k)$ with respect to the aspect ratio $(a / b)$ for three layered cross-ply [0/90/0] composite SSSS skew plates $\left(\psi=30^{\circ}, a / h=100\right)$ subjected to different in-plane loadingcboxs.

\section{CONCLUSIONS}

The pre-buckling vibration and buckling behaviour of composite skew plates with different skew angles and boundary conditions have been investigated under different types of linearly varying in-plane loadings. The present analysis is based on higher-order shear deformation theory (HSDT) for determining more realistic behaviour of composite skew plates. The total energy functional is derived using in-plane stress distributions and transformed from the physical domain to the computational domain using transformation relations. This functional is solved using the Rayleigh-Ritz method (RRM) considering boundary characteristics orthonormal polynomials (BCOPs) functions. The pre-buckled free vibration frequency and critical buckling loads for composite skew plates under different linearly varying in-plane loadings are obtained from the solution of the associated linear eigenvalue problem. The free vibration frequency of the skew plate under both loaded and unloaded conditions increases with an increase in edge restraint and skew angle. For different types of linearly varying in-plane loading, the pre-buckling fundamental frequency increases with an increase in the $\gamma$ value. For all types of linearly varying in-plane loading in static stability analysis, the value of the buckling coefficient decreases after shifting the buckling mode from first mode to second mode. With the reduction of length to thickness ratio (thicker plate), the free vibration 
Table 3. Dimensionless buckling coefficient $\left(k_{c}\right)$ for cross-ply [0/90/0] composite skew plates $\left(\psi=45^{\circ}, a / h=100\right)$ with nine sets of boundary conditions subjected to trapezoidal $(\gamma=0.5)$ in-plane loading.

\begin{tabular}{||c|c|c|c|c|c|c|c|c|c|}
\hline Aspect ratio & SSSS & SSCS & SCSS & CSCS & SCSC & SSCC & CCSC & CCCS & CCCC \\
\hline 0.5 & 12.85 & 23.67 & 13.49 & 43.19 & 13.97 & 23.83 & 24.67 & 43.27 & 44.29 \\
\hline 0.75 & 7.44 & 12.50 & 8.46 & 21.56 & 9.48 & 12.87 & 14.17 & 21.88 & 22.87 \\
\hline 1.0 & 5.74 & 8.70 & 7.31 & 13.95 & 9.11 & 9.36 & 11.29 & 15.10 & 15.59 \\
\hline 1.25 & 5.26 & 7.18 & 7.63 & 10.55 & 10.47 & 8.24 & 10.92 & 12.08 & 12.67 \\
\hline 1.5 & 5.43 & 6.70 & 8.76 & 8.93 & 10.55 & 8.27 & 11.03 & 11.17 & 11.74 \\
\hline 1.75 & 6.05 & 6.88 & 9.25 & 8.29 & 10.69 & 8.78 & 10.81 & 11.03 & 11.98 \\
\hline 2.0 & 6.71 & 7.55 & 9.79 & 8.29 & 11.67 & 8.80 & 12.02 & 12.12 & 12.60 \\
\hline
\end{tabular}

Table 4. Dimensionless buckling coefficient $\left(k_{c}\right)$ for cross-ply [0/90/0] composite skew plates $\left(\psi=45^{\circ}, a / h=100\right)$ with nine sets of boundary conditions subjected to trapezoidal $(\gamma=0.5)$ in-plane loading.

\begin{tabular}{||c|c|c|c|c|c|c|c|c|c|}
\hline \hline Aspect ratio & SSSS & SSCS & SCSS & CSCS & SCSC & SSCC & CCSC & CCCS & CCCC \\
\hline 0.5 & 14.70 & 26.66 & 15.76 & 47.47 & 16.12 & 26.70 & 28.11 & 49.89 & 49.98 \\
\hline 0.75 & 9.40 & 15.50 & 11.21 & 25.68 & 12.13 & 15.68 & 17.77 & 28.77 & 28.85 \\
\hline 1.0 & 8.25 & 12.26 & 11.45 & 18.53 & 13.26 & 12.69 & 15.86 & 22.76 & 23.10 \\
\hline 1.25 & 8.93 & 12.03 & 14.64 & 16.14 & 16.91 & 12.80 & 17.44 & 22.36 & 23.04 \\
\hline 1.5 & 11.20 & 14.09 & 19.06 & 16.27 & 19.91 & 15.19 & 20.83 & 25.34 & 25.80 \\
\hline 1.75 & 14.17 & 17.23 & 25.09 & 18.07 & 25.22 & 17.25 & 25.73 & 30.55 & 30.65 \\
\hline 2.0 & 17.92 & 17.87 & 18.00 & 20.64 & 32.36 & 19.91 & 32.78 & 35.12 & 35.73 \\
\hline
\end{tabular}

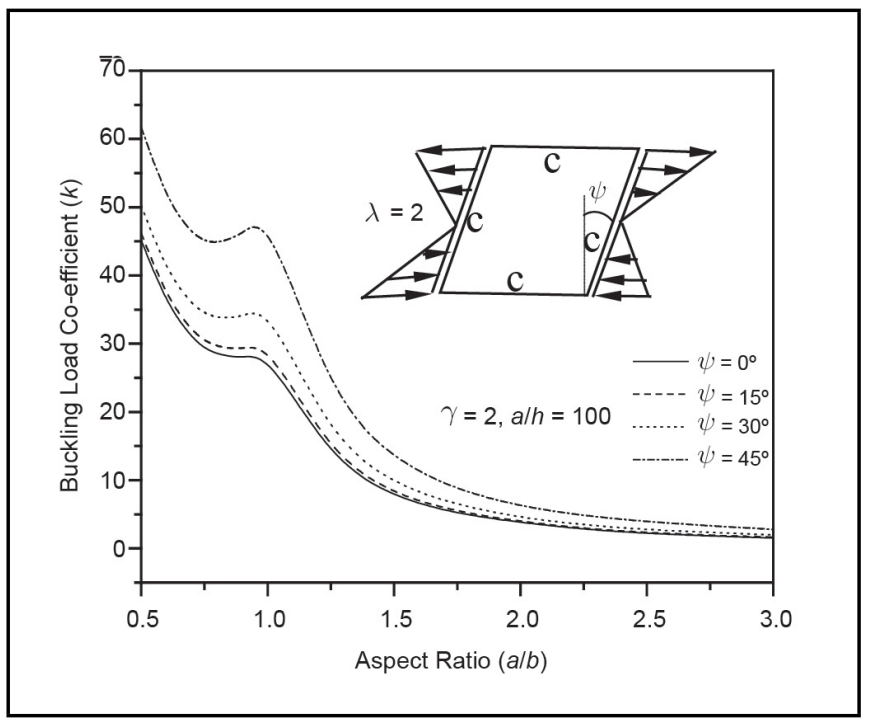

Figure 9. Variation of buckling load coefficient $(k)$ with respect to the aspect ratio $(a / b)$ for three layered cross-ply [0/90/0] composite CCCC skew plates having different skew angles subjected to in-plane bending.

frequencies decrease with an increase in the aspect ratio. It is also noted that the more edge restraint there is, the higher the buckling coefficient is.

\section{APPENDICES}

\section{Appendix A}

$$
\begin{gathered}
\Re_{1}\left(u_{, \xi}^{o}, u_{, \eta}^{o}, v_{, \xi}^{o}, v_{, \eta}^{o}\right)=\frac{1}{a^{2}}\left(A_{11}+A_{66} \tan ^{2} \psi\right)\left(u_{, \xi}^{o}\right)^{2}- \\
\frac{2}{a b} A_{66} \tan \psi \sec \psi u_{, \xi}^{o} u_{, \eta}^{o}+\frac{1}{b^{2}} A_{66} \sec ^{2} \psi\left(u_{, \eta}^{o}\right)^{2}- \\
\frac{2}{a^{2}}\left(A_{11}+A_{66}\right) \tan \psi u_{, \xi}^{o} v_{, \xi}^{o}+\frac{2}{a b} A_{12} \sec \psi u_{, \xi}^{o} v_{, \eta}^{o}+ \\
\frac{2}{a b} A_{66} \sec \psi u_{, \eta}^{o} v_{, \xi}^{o}+\frac{1}{a^{2}}\left(A_{22} \tan ^{2} \psi+A_{66}\right)\left(v_{, \xi}^{o}\right)^{2}- \\
\frac{2}{a b} A_{22} \tan \psi \sec \psi v_{, \xi}^{o} v_{, \eta}^{o}+\frac{1}{b^{2}} A_{22} \sec ^{2} \psi\left(v_{, \eta}^{o}\right)^{2}
\end{gathered}
$$

$\Re_{2}\left(w_{, \xi \xi}^{o}, w_{, \xi \eta}^{o}, w_{, \eta \eta}^{o}, \phi_{1, \xi}^{o}, \phi_{1, \eta}^{o}, \phi_{2, \xi}^{o}, \phi_{2, \eta}^{o}\right)=\frac{1}{a^{4}}\left(D_{11}+\right.$

$\left.2 D_{12} \tan ^{2} \psi+4 D_{66} \tan ^{2} \psi+D_{22} \tan ^{4} \psi\right)\left(w_{, \xi \xi}^{o}\right)^{2}-$

$\frac{4}{a^{3} b}\left(D_{12}+2 D_{66}+D_{22} \tan ^{2} \psi\right) \tan \psi \sec \psi w_{, \xi \xi}^{o} w_{, \xi \eta}^{o}+$

$\frac{2}{a^{2} b^{2}}\left(D_{12}+D_{22} \tan ^{2} \psi\right) \sec ^{2} \psi w_{, \xi \xi}^{o} w_{, \eta \eta}^{o}+$

$\frac{4}{a^{2} b^{2}}\left(D_{66}+D_{22} \tan ^{2} \psi\right) \sec ^{2} \psi\left(w_{, \xi \eta}^{o}\right)^{2}-$

$\frac{4}{a b^{3}} D_{22} \tan \psi \sec ^{3} \psi w_{, \xi \eta}^{o} w_{, \eta \eta}^{o}+\frac{1}{b^{4}} D_{22} \sec ^{4} \psi\left(w_{, \eta \eta}^{o}\right)^{2}-$

$\frac{1}{a^{3}}\left(E_{11}+E_{12} \tan ^{2} \psi+2 E_{66} \tan ^{2} \psi\right) w_{, \xi \xi}^{o} \phi_{1, \xi}^{o}+$

$\frac{2}{a^{2} b} E_{66} \tan \psi \sec \psi w_{, \xi \xi}^{o} \phi_{1, \eta}^{o}+\frac{1}{a^{3}}\left(E_{12}+2 E_{66}+\right.$

$\left.E_{22} \tan ^{2} \psi\right) \tan \psi w_{, \xi \xi}^{o} \phi_{2, \xi^{-}}^{o}$

$\frac{1}{a^{2} b}\left(E_{12}+E_{22} \tan ^{2} \psi\right) \sec \psi w_{, \xi \xi}^{o} \phi_{2, \eta}^{o}+$

$\frac{2}{a^{2} b}\left(E_{12}+E_{66}\right) \tan \psi \sec \psi w_{, \xi \eta}^{o} \phi_{1, \xi}^{o}$

$\frac{2}{a b^{2}} E_{66} \sec ^{2} \psi w_{, \xi \eta}^{o} \phi_{1, \eta}^{o}-$

$\frac{2}{a^{2} b}\left(E_{66}+E_{22} \tan ^{2} \psi\right) \sec \psi w_{, \xi \eta}^{o} \phi_{2, \xi}^{o}+$

$\frac{2}{a b^{2}} E_{22} \tan \psi \sec ^{2} \psi w_{, \xi \eta}^{o} \phi_{2, \eta}^{o}-$

$\frac{1}{a b^{2}} E_{12} \sec ^{2} \psi w_{, \eta \eta}^{o} \phi_{1, \xi}^{o}+$

$\frac{1}{a b^{2}} E_{22} \tan \psi \sec ^{2} \psi w_{, \eta \eta}^{o} \phi_{2, \xi}^{o}-$

$\frac{1}{b^{3}} E_{22} \sec ^{3} \psi w_{, \eta \eta}^{o} \phi_{2, \eta}^{o}$

$$
\begin{aligned}
& \Re_{3}\left(w_{, \xi \xi}^{o}, w_{, \xi \eta}^{o}, w_{, \eta \eta}^{o}, \phi_{1, \xi}^{o}, \phi_{1, \eta}^{o}, \phi_{2, \xi}^{o}, \phi_{2, \eta}^{o}\right)= \\
& -\frac{1}{a^{3}}\left(E_{11}+E_{12} \tan ^{2} \psi+2 E_{66} \tan ^{2} \psi\right) w_{, \xi \xi}^{o} \phi_{1, \xi}^{o}+ \\
& \frac{2}{a^{2} b} E_{66} \tan \psi \sec \psi w_{, \xi \xi}^{o} \phi_{1, \eta}^{o}+
\end{aligned}
$$


$\overline{\text { Table 5. Dimensionless buckling coefficient }\left(k_{c}\right) \text { for three layered cross-ply [0/90/0] composite skew plates }\left(\psi=45^{\circ}, a / h=100\right) \text { with nine sets of boundary }}$ conditions subjected to partial tension $(\gamma=1.5)$ in-plane loading.

\begin{tabular}{||c|c|c|c|c|c|c|c|c|c|}
\hline \hline Aspect ratio & SSSS & SSCS & SCSS & CSCS & SCSC & SSCC & CCSC & CCCS & CCCC \\
\hline 0.5 & 16.86 & 29.81 & 18.60 & 51.66 & 18.83 & 29.79 & 32.05 & 55.22 & 55.59 \\
\hline 0.75 & 12.23 & 19.32 & 15.76 & 30.28 & 16.36 & 19.35 & 22.91 & 35.32 & 35.99 \\
\hline 1.0 & 12.97 & 18.05 & 21.00 & 24.62 & 21.76 & 18.14 & 24.05 & 33.18 & 33.73 \\
\hline 1.25 & 17.82 & 22.34 & 25.99 & 25.24 & 29.96 & 22.36 & 31.20 & 34.82 & 39.34 \\
\hline 1.5 & 12.53 & 12.96 & 13.08 & 16.76 & 21.54 & 21.98 & 25.25 & 16.98 & 26.95 \\
\hline 1.75 & 7.59 & 7.73 & 8.19 & 9.85 & 13.42 & 13.21 & 15.35 & 10.35 & 16.04 \\
\hline 2.0 & 5.28 & 5.36 & 5.74 & 6.59 & 9.49 & 9.09 & 10.69 & 7.13 & 10.89 \\
\hline
\end{tabular}

$\frac{1}{a^{3}}\left(E_{12}+2 E_{66}+E_{22} \tan ^{2} \psi\right) \tan \psi w_{, \xi \xi}^{o} \phi_{2, \xi}^{o}-$

$\frac{1}{a^{2} b}\left(E_{12}+E_{22} \tan ^{2} \psi\right) \sec \psi w_{, \xi \xi}^{o} \phi_{2, \eta}^{o}+$

$\frac{2}{a^{2} b}\left(E_{12}+E_{66}\right) \tan \psi \sec \psi w_{, \xi \eta}^{o} \phi_{1, \xi}^{o}$

$\frac{2}{a b^{2}} E_{66} \sec ^{2} \psi w_{, \xi \eta}^{o} \phi_{1, \eta}^{o}-$

$\frac{2}{a^{2} b}\left(E_{66}+E_{22} \tan ^{2} \psi\right) \sec \psi w_{, \xi \eta}^{o} \phi_{2, \xi}^{o}+$

$\frac{2}{a b^{2}} E_{22} \tan \psi \sec ^{2} \psi w_{, \xi \eta}^{o} \phi_{2, \eta}^{o}-$

$\frac{1}{a b^{2}} E_{12} \sec ^{2} \psi w_{, \eta \eta}^{o} \phi_{1, \xi}^{o}+$

$\frac{1}{a b^{2}} E_{22} \tan \psi \sec ^{2} \psi w_{, \eta \eta}^{o} \phi_{2, \xi}^{o}-$

$\frac{1}{b^{3}} E_{22} \sec ^{3} \psi w_{, \eta \eta}^{o} \phi_{2, \eta}^{o}+$

$\frac{1}{a^{2}}\left(F_{11}+F_{66} \tan ^{2} \psi\right)\left(\phi_{1, \xi}^{o}\right)^{2}-$

$\frac{2}{a b} F_{66} \tan \psi \sec \psi \phi_{1, \xi}^{o} \phi_{1, \eta}^{o}+$

$\frac{1}{b^{2}} F_{66} \sec ^{2} \psi\left(\phi_{1, \eta}^{o}\right)^{2}-$

$\frac{2}{a^{2}}\left(F_{11}+F_{66}\right) \tan \psi \phi_{1, \xi}^{o} \phi_{2, \xi}^{o}+$

$\frac{2}{a b} F_{12} \sec \psi \phi_{1, \xi}^{o} \phi_{2, \eta}^{o}+$

$\frac{2}{a b} F_{66} \sec \psi \phi_{1, \eta}^{o} \phi_{2, \xi}^{o}+$

$\frac{1}{a^{2}}\left(F_{22} \tan ^{2} \psi+A_{66}\right)\left(\phi_{2, \xi}^{o}\right)^{2}-$

$\frac{2}{a b} F_{22} \tan \psi \sec \psi \phi_{2, \xi}^{o} \phi_{2, \eta}^{o}+\frac{1}{b^{2}} F_{22} \sec ^{2} \psi\left(\phi_{2, \eta}^{o}\right)^{2}$

$$
\Re_{4}\left(\phi_{1}^{o}, \phi_{2}^{o}\right)=H_{44}\left(\phi_{2}^{o}\right)^{2}+H_{55}\left(\phi_{1}^{o}\right)^{2}
$$

$$
\begin{aligned}
& \Re_{5}\left(n_{x x}, n_{x y}, n_{y y}, w_{, \xi}^{o}, w_{, \eta}^{o}\right)= \\
& \left(\frac{n_{x x}}{a^{2}}-\frac{2 n_{x y}}{a b} \tan \psi+\frac{n_{y y}}{b^{2}} \tan ^{2} \psi\right)\left(w_{, \xi}^{o}\right)^{2}+ \\
& \left(\frac{2 n_{x y}}{a b} \sec \psi-\frac{2 n_{y y}}{b^{2}} \tan \psi \sec \psi\right) w_{, \xi}^{o} w_{, \eta}^{o}+ \\
& \frac{n_{y y}}{b^{2}} \sec ^{2} \psi\left(w_{, \eta}^{o}\right)^{2}
\end{aligned}
$$

$$
\begin{aligned}
& \Re_{6}\left(u_{, t}^{o}, v_{, t}^{o}, w_{, t}^{o}, \phi_{1, t}^{o}, \phi_{2, t}^{o}\right)= \\
& \rho h\left[\left(u_{, t}^{o}\right)^{2}+\left(v_{, t}^{o}\right)^{2}+\left(w_{, t}^{o}\right)^{2}\right]+ \\
& \frac{\rho h^{3}}{12}\left[\left(\phi_{1, t}^{o}\right)^{2}+\left(\phi_{2, t}^{o}\right)^{2}\right]
\end{aligned}
$$

\section{Appendix B}

$$
\begin{aligned}
\Theta_{11}= & 30 \sqrt{182}(-1+\eta) \eta \xi\left(-1+13 \xi-57 \xi^{2}+\right. \\
& \left.111 \xi^{3}-99 \xi^{4}+33 \xi^{5}\right)
\end{aligned}
$$

$$
\begin{aligned}
\Theta_{12}= & 210 \sqrt{11} \eta\left(1-3 \eta+2 \eta^{2}\right) \xi(1-9 \xi+ \\
& \left.26 \xi^{2}-30 \xi^{3}+12 \xi^{4}\right)
\end{aligned}
$$

$$
\begin{aligned}
\Theta_{13}= & 90 \eta\left(-3+17 \eta-28 \eta^{2}+14 \eta^{3}\right) \xi(-3+17 \xi- \\
& \left.28 \xi^{2}+14 \xi^{3}\right)
\end{aligned}
$$

$$
\begin{aligned}
\Theta_{14}= & 210 \sqrt{11} \eta\left(1-9 \eta+26 \eta^{2}-\right. \\
& \left.30 \eta^{3}+12 \eta^{4}\right) \xi\left(1-3 \xi+2 \xi^{2}\right)
\end{aligned}
$$

$$
\begin{aligned}
\Theta_{15}= & 30 \sqrt{182} \eta\left(-1+13 \eta-57 \eta^{2}+\right. \\
& \left.111 \eta^{3}-99 \eta^{4}+33 \eta^{5}\right) \xi(-1+\xi)
\end{aligned}
$$

$\Theta_{16}=30 \sqrt{42}(-1+\eta) \eta \xi(3-53 \xi+$

$$
\left.325 \xi^{2}-935 \xi^{3}+1375 \xi^{4}-1001 \xi^{5}+286 \xi^{6}\right)
$$

$$
\begin{aligned}
\Theta_{17}= & 210 \sqrt{26} \eta\left(1-3 \eta+2 \eta^{2}\right) \xi \\
& \left(-1+13 \xi-57 \xi^{2}+111 \xi^{3}-99 \xi^{4}+33 \xi^{5}\right)
\end{aligned}
$$

$$
\begin{aligned}
\Theta_{18}= & 30 \sqrt{231} \eta\left(-3+17 \eta-28 \eta^{2}\right. \\
& \left.+14 \eta^{3}\right) \xi\left(1-9 \xi+26 \xi^{2}-30 \xi^{3}+12 \xi^{4}\right)
\end{aligned}
$$




$$
\begin{aligned}
\Theta_{19}= & 30 \sqrt{231} \eta\left(1-9 \eta+26 \eta^{2}-\right. \\
\left.30 \eta^{3}+12 \eta^{4}\right) \xi\left(-3+17 \xi-28 \xi^{2}+14 \xi^{3}\right) & \\
\Theta_{20}= & 210 \sqrt{26} \eta\left(-1+13 \eta-57 \eta^{2}+\right. \\
& \left.111 \eta^{3}-99 \eta^{4}+33 \eta^{5}\right) \xi\left(1-3 \xi+2 \xi^{2}\right) \\
\Theta_{21}= & 30 \sqrt{42} \eta\left(3-53 \eta+325 \eta^{2}-\right. \\
& \left.935 \eta^{3}+1375 \eta^{4}-1001 \eta^{5}+286 \eta^{6}\right) \xi(-1+\xi) \\
\Theta_{22}= & 30 \sqrt{714}(-1+\eta) \eta \xi\left(-1+23 \xi-187 \xi^{2}+\right. \\
& \left.737 \xi^{3}-1573 \xi^{4}+1859 \xi^{5}-1144 \xi^{6}+286 \xi^{7}\right), \\
\Theta_{23}= & 210 \sqrt{6}\left(1-3 \eta+2 \eta^{2}\right) \eta \xi(3-53 \xi+ \\
& \left.325 \xi^{2}-935 \xi^{3}+1375 \xi^{4}-1001 \xi^{5}+286 \xi^{6}\right), \\
\Theta_{24}= & 30 \sqrt{546}\left(-3+17 \eta-28 \eta^{2}+14 \eta^{3}\right) \\
& \eta \xi\left(-1+13 \xi-57 \xi^{2}+111 \xi^{3}-99 \xi^{4}+33 \xi^{5}\right),
\end{aligned}
$$

$$
\begin{gathered}
\Theta_{25}=2310 \eta\left(1-9 \eta+26 \eta^{2}-30 \eta^{3}+\right. \\
\left.12 \eta^{4}\right) \xi\left(1-9 \xi+26 \xi^{2}-30 \xi^{3}+12 \xi^{4}\right) \\
\Theta_{26}=30 \sqrt{546} \eta\left(-1+13 \eta-57 \eta^{2}+111 \eta^{3}-\right. \\
\left.99 \eta^{4}+33 \eta^{5}\right) \xi\left(-3+17 \xi-28 \xi^{2}+14 \xi^{3}\right),
\end{gathered}
$$

$$
\begin{aligned}
\Theta_{27}= & 210 \sqrt{6} \eta\left(3-53 \eta+325 \eta^{2}-935 \eta^{3}+\right. \\
& \left.1375 \eta^{4}-1001 \eta^{5}+286 \eta^{6}\right) \xi\left(1-3 \xi+2 \xi^{2}\right)
\end{aligned}
$$

$$
\begin{aligned}
\Theta_{28}= & 30 \sqrt{714} \eta\left(-1+23 \eta-187 \eta^{2}+737 \eta^{3}-\right. \\
& \left.1573 \eta^{4}+1859 \eta^{5}-1144 \eta^{6}+286 \eta^{7}\right)(-1+\xi),
\end{aligned}
$$

$\Theta_{29}=30 \sqrt{1254}(-1+\eta) \eta \xi\left(1-29 \xi+301 \xi^{2}-1547 \xi^{3}+\right.$ $\left.4459 \xi^{4}-7553 \xi^{5}+7462 \xi^{6}-3978 \xi^{7}+884 \xi^{8}\right)$,

$$
\begin{aligned}
\Theta_{30}= & 210 \sqrt{102} \eta\left(1-3 \eta+2 \eta^{2}\right) \xi\left(-1+23 \xi-187 \xi^{2}+\right. \\
& \left.737 \xi^{3}-1573 \xi^{4}+1859 \xi^{5}-1144 \xi^{6}+286 \xi^{7}\right),
\end{aligned}
$$

$$
\begin{aligned}
\Theta_{31}= & 90 \sqrt{14} \eta\left(-3+17 \eta-28 \eta^{2}+14 \eta^{3}\right) \xi(3-53 \xi+ \\
& \left.325 \xi^{2}-935 \xi^{3}+1375 \xi^{4}-1001 \xi^{5}+286 \xi^{6}\right),
\end{aligned}
$$

$$
\begin{aligned}
\Theta_{32}= & 210 \sqrt{286} \eta\left(1-9 \eta+26 \eta^{2}-30 \eta^{3}+12 \eta^{4}\right) \\
& \xi\left(-1+13 \xi-57 \xi^{2}+111 \xi^{3}-99 \xi^{4}+33 \xi^{5}\right),
\end{aligned}
$$

$$
\begin{aligned}
\Theta_{33}= & 210 \sqrt{286} \eta\left(-1+13 \eta-57 \eta^{2}+111 \eta^{3}-99 \eta^{4}+\right. \\
& \left.33 \eta^{5}\right) \xi\left(1-9 \xi+26 \xi^{2}-30 \xi^{3}+12 \xi^{4}\right),
\end{aligned}
$$

$$
\begin{gathered}
\Theta_{34}=90 \sqrt{14} \eta\left(3-53 \eta+325 \eta^{2}-935 \eta^{3}+1375 \eta^{4}-\right. \\
\left.1001 \eta^{5}+286 \eta^{6}\right) \xi\left(-3+17 \xi-28 \xi^{2}+14 \xi^{3}\right),
\end{gathered}
$$

$$
\begin{aligned}
\Theta_{35}= & 30 \sqrt{1122 / 1303}(-1+\eta) \eta \xi \\
& \left(1-3 \xi+2 \xi^{2}\right)\left(-31-154 \eta+1155 \eta^{2}-4004 \eta^{3}+\right. \\
& 7007 \eta^{4}-6006 \eta^{5}+2002 \eta^{6}+988 \xi-8398 \xi^{2}+ \\
& \left.31616 \xi^{3}-57798 \xi^{4}+50388 \xi^{5}-16796 \xi^{6}\right)
\end{aligned}
$$

$$
\begin{aligned}
\Theta_{36}= & 30 \sqrt{1254 / 417437}(-1+\eta) \eta(-1+\xi) \xi(635+308 \eta- \\
& 3003 \eta^{2}+14014 \eta^{3}-35035 \eta^{4}+48048 \eta^{5}-34034 \eta^{6}+ \\
& 9724 \eta^{7}-18088 \xi+176358 \xi^{2}-823004 \xi^{3}+ \\
& \left.2057510 \xi^{4}-2821728 \xi^{5}+1998724 \xi^{6}-571064 \xi^{7}\right)
\end{aligned}
$$

\section{REFERENCES}

${ }^{1}$ Durvasula, S. Buckling of simply supported skew plates, $J$. Eng. Mech., 97 (3), 967-979, (1971).

2 Liew, K.M. and Lam, K.Y. Application of two-dimensional orthogonal plate function to flexural vibration of skew plates, J. Sound. Vib, 139 (2), 241-252, (1990). https://dx.doi.org/10.1016/0022-460X(90)90885-4

3 Wang, C. M., Liew, K. M., and Alwis, W. A. M. Buckling of skew plates and corner condition for simply supported edges, J. Eng. Mech., 118 (4), 651-662, (1992). https://dx.doi.org/10.1061/(ASCE)07339399(1992)118:4(651)

${ }^{4}$ Singh, B. and Chakraverty, S. Flexural vibration of skew plates using boundary characteristic orthogonal polynomials in two variables, J. Sound. Vib., 173 (2), 157-178, (1994). https://dx.doi.org/10.1006/jsvi.1994.1224

5 Wang, S. Buckling analysis of skew fibre-reinforced composite laminates based on first-order shear deformation plate theory, Compos. Struct., 37 (1), 5-19, (1997). https://dx.doi.org/10.1016/S0263-8223(97)00050-0

${ }^{6}$ Mizusawa, T., Kajita, T. and Naruoka, M. Buckling of skew plate structures using B-spline functions, Int. J. Numer. Meth. Eng., 15 (1), 87-96, (1980). https://dx.doi.org/doi.org/10.1002/nme.1620150108

7 Taj, G. and Chakrabarti, A. Static and dynamic analysis of functionally graded skew plates, J. Eng. Mech., 139 (7), 848-857, (2012).https://dx.doi.org/10.1061/(ASCE)EM.19437889.0000523 
8 Kitipornchai, S., Xiang, Y., Wang, C. M. and Liew, K. M. Buckling of thick skew plates, Int. J. Numer. Meth. Eng., 36 (8), 1299-1310, (1993). https://dx.doi.org/10.1002/nme.1620360804

9 Farag, A. M. and Ashour, A. S. Free vibration of orthotropic skew plates, J. Vib. Acoust., 122 (3), 313-317, (2000). https://dx.doi.org/10.1115/1.1302085

10 Ganapathi, M. and Prakash, T. Thermal buckling of simply supported functionally graded skew plates, Compos. Struct., 74 (2), 247-250, (2006).https://dx.doi.org/10.1016/j.compstruct.2005.04.004

11 Wang, X., Tan, M., and Zhou, Y. Buckling analyses of anisotropic plates and isotropic skew plates by the new version differential quadrature method, Thin Wall Struct., 41 (1), 15-29, (2003). https://dx.doi.org/10.1016/S02638231(02)00100-3

12 Daripa, R. and Singha, M. K. Influence of corner stresses on the stability characteristics of composite skew plates, Int. J. Nonlinear Mech., 44 (2), 138-146, (2009). https://dx.doi.org/10.1016/j.ijnonlinmec.2008.10.003

13 Karami, G., Ali Shahpari, S., and Malekzadeh, P. DQM analysis of skewed and trapezoidal laminated plates, Compos. Struct., 59 (3), 393-402, (2003). https://dx.doi.org/10.1016/S0263-8223(02)00188-5

14 Zhou, L. and Zheng, W. X. Vibration of skew plates by the MLS-Ritz method, Int. J. Mech. Sci., 50 (7), 1133-1141, (2008). https://dx.doi.org/10.1016/j.ijmecsci.2008.05.002

15 Singha, M. K. and Ganapathi, M. Large amplitude free flexural vibrations of laminated composite skew plates, Int. J. Nonlin. Mech., 39 (10), 1709-1720, (2004). https://dx.doi.org/10.1016/j.ijnonlinmec.2004.04.003

16 Eftekhari, S. A. and Jafari, A. A. Modified mixed RitzDQ formulation for free vibration of thick rectangular and skew plates with general boundary conditions, Appl. Math Model., 37 (12-13), 7398-7426, (2013). https://dx.doi.org/10.1016/j.apm.2013.02.040

17 Wang, X., Wang, Y., and Yuan, Z. Accurate vibration analysis of skew plates by the new version of the differential quadrature method, Appl. Math Model., 38 (3), 926-937, (2014). https://dx.doi.org/10.1016/j.apm.2013.07.021

18 Leissa, A. W. and Kang, J. H. Exact solutions for vibration and buckling of an SS-C-SS-C rectangular plate loaded by linearly varying in-plane stresses, Int. J. Mech. Sci., 44 (9), 1925-1945, (2002). https://dx.doi.org/10.1016/S00207403(02)00069-3

19 Kang, J. H. and Leissa, A. W. Vibration and buckling of SS-F-SS-F rectangular plates loaded by in-plane moments, Int. J. Struct. Stab. Dy., 1 (4), 527-543, (2001). https://dx.doi.org/10.1142/S0219455401000299

20 Kang, J. H. and Leissa, A. W. Exact solutions for the buckling of rectangular plates having linearly varying in-plane loading on two opposite simply supported edges, Int. J. Solids Struct., 42 (14), 4220-4238, (2005). https://dx.doi.org/10.1016/j.ijsolstr.2004.12.011
21 Zhong, H. and Gu, C. Buckling of symmetrical crossply composite rectangular plates under a linearly varying in-plane load, Compos. Struct., 80 (1), 42-48, (2007). https://dx.doi.org/10.1016/j.compstruct.2006.02.030

22 Lai, S. K. and Xiang, Y. DSC analysis for buckling and vibration of rectangular plates with elastically restrained edges and linearly varying in-plane loading, Int. J. Struct. Stab. Dy., 9 (3), 511-531, (2009). https://dx.doi.org/doi.org/10.1142/S0219455409003119

23 Lopatin, A. V. and Morozov, E. V. Buckling of the SSCF rectangular orthotropic plate subjected to linearly varying in-plane loading, Compos. Struct., 93 (7), 1900-1909, (2011).https://dx.doi.org/10.1016/j.compstruct.2011.01.024

24 Wang, X., Wang, X., and Shi, X. Differential quadrature buckling analyses of rectangular plates subjected to non-uniform distributed in-plane loadings, Thin Wall Struct., 44 (8), 837-843, (2006). https://dx.doi.org/10.1016/j.tws.2006.08.008

25 Wang, X., Wang, X., and Shi, X. Accurate buckling loads of thin rectangular plates under parabolic edge compressions by the differential quadrature method, Int. J. Mech. Sci., 49 (4), 447-453, (2007). https://dx.doi.org/10.1016/j.ijmecsci.2006.09.004

26 Wang, X., Gan, L., and Wang, Y. A differential quadrature analysis of vibration and buckling of an SS-C-SS$\mathrm{C}$ rectangular plate loaded by linearly varying in-plane stresses, J. Sound Vib., 298 (1-2), 420-431, (2006). https://dx.doi.org/doi.org/10.1016/j.jsv.2006.06.003

27 Tang, Y. and Wang, X. Buckling of symmetrically laminated rectangular plates under parabolic edge compressions, Int. J. Mech. Sci., 53 (2), 91-97, (2011).https://dx.doi.org/10.1016/j.ijmecsci.2010.11.005

28 Kumar, R., Kumar, A. and Panda, S. K. Parametric resonance of composite skew plate under non-uniform inplane loading, Struct. Eng. Mech., 55 (2), 435-459, (2015). https://dx.doi.org/10.12989/sem.2015.55.2.435

29 Reddy, J. N. and Liu, C. F. A higher-order shear deformation theory of laminated elastic shells, Int. J. Eng. Sci., 23 (3), 319-330, (1985). https://dx.doi.org/10.1016/00207225(85)90051-5

30 Soldatos, K. P. A refined laminated plate and shell theory with applications, J. Sound Vib., 144 (1), 109-129, (1991). https://dx.doi.org/10.1016/0022-460X(91)90736-4

31 Nair, P. S. and Durvasula, S. Vibration of skew plates, J. Sound Vib., 26 (1), 1-19, (1973). https://dx.doi.org/10.1016/S0022-460X(73)80201-9

32 Babu, C. S. and Kant, T. Two shear deformable finite element models for buckling analysis of skew fibre-reinforced composite and sandwich panels, Compos. Struct., 46 (2), 115-124, (1999). https://dx.doi.org/10.1016/S02638223(99)00039-2

33 Durvasula, S. Buckling of clamped skew plates, AIAA. J., 8 (1), 178-181, (1970). https://dx.doi.org/doi.org/10.2514/3.5635 